\title{
Excitation Functions of Related Parameters from Transverse Momentum (Mass) Spectra in High-Energy Collisions
}

\author{
Li-Li Li, ${ }^{1}$ Fu-Hu Liu $\mathbb{D}^{1}{ }^{1}$ Muhammad Waqas $\mathbb{D}^{1},{ }^{1}$ Rasha Al-Yusufi, ${ }^{1,2}$ and Altaf Mujear ${ }^{1,2}$ \\ ${ }^{1}$ Institute of Theoretical Physics and State Key Laboratory of Quantum Optics and Quantum Optics Devices, Shanxi University, \\ Taiyuan, Shanxi 030006, China \\ ${ }^{2}$ Physics Department, Faculty of Science, Sana'a University, P. O. Box 13499, Sana'a, Yemen
}

Correspondence should be addressed to Fu-Hu Liu; fuhuliu@163.com

Received 29 January 2020; Accepted 6 May 2020; Published 10 June 2020

Academic Editor: Torsten Asselmeyer-Maluga

Copyright (c) $2020 \mathrm{Li}-\mathrm{Li} \mathrm{Li}$ et al. This is an open access article distributed under the Creative Commons Attribution License, which permits unrestricted use, distribution, and reproduction in any medium, provided the original work is properly cited. The publication of this article was funded by $\mathrm{SCOAP}^{3}$.

Transverse momentum (mass) spectra of positively and negatively charged pions and of positively and negatively charged kaons, protons, and antiprotons produced at mid-(pseudo)rapidity in various collisions at high energies are analyzed in this work. The experimental data measured in central gold-gold, central lead-lead, and inelastic proton-proton collisions by several international collaborations are studied. The (two-component) standard distribution is used to fit the data and extract the excitation function of effective temperature. Then, the excitation functions of kinetic freeze-out temperature, transverse flow velocity, and initial temperature are obtained. In the considered collisions, the four parameters increase with the increase of collision energy in general, and the kinetic freeze-out temperature appears at the trend of saturation at the top Relativistic Heavy Ion Collider and the Large Hadron Collider.

\section{Introduction}

It is believed that the environment of high temperature and high density is formed in the system evolution process of central nucleus-nucleus (AA) collisions at high energy [1-3], in which quark-gluon plasma (QGP) is possibly created and many particles are produced [4-6]. At present, it is impossible to detect directly the system evolution process of collisions due to very short time interval. Instead, the particle spectra at the stage of kinetic freeze-out can be measured in experiments and the mechanisms of system evolutions and particle productions can be studied indirectly [7-9], though the particle ratios reflect the property at the stage of chemical freeze-out. As for peripheral AA collisions and small collision system, the situation is similar if the multiplicity is high enough due to the small system which also appears collective behavior $[10,11]$.

Although there are different stages in the system evolution [1-3], the initial state is the most important due to its determining effect to the system evolution. In addition, chemical and kinetic freeze-outs are two important stages in the system evolution. At the stage of chemical freeze-out, the system had a phase transition from QGP to hadronic matter, and the constituents and ratios of various particles do not change anymore. At the stage of kinetic freeze-out, the collisions among various particles are elastic, and the transverse momentum spectra of various particles are fixed [2, 7]. In the small system with low multiplicity, QGP is not expected to create in it due to a very small volume of the violent collision region. From the similar multiplicity at the energy up to $200 \mathrm{GeV}$, the small system is more similar to peripheral AA collisions, but not to central AA collisions [12, 13]. At the energy down to 10 or several $\mathrm{GeV}$, the situation is different due to the fact that the baryon-dominated effect plays more important role in AA collisions [14].

The temperatures at the stages of kinetic freeze-out, chemical freeze-out, and initial state are called the kinetic freeze-out temperature ( $T_{0}$ or $\left.T_{\text {kin }}\right)$, chemical freeze-out temperature $\left(T_{\mathrm{ch}}\right)$, and initial temperature $\left(T_{\mathrm{i}}\right)$, respectively. Besides, one also has the effective temperature $(T)$ in which both the contributions of thermal motion and flow effect 
are included. It is expected that various temperatures can be extracted from particle spectra, which are usually model dependent. Generally, $T$ is unavoidably model dependent, and $T_{\mathrm{ch}}$ extracted from particle ratios in the statistical thermal model [15-18] is also model dependent. We hope to use a less model-dependent method to extract $T_{0}, \beta_{\mathrm{T}}$, and $T_{\mathrm{i}}$. The quantities used in the method are expected to relate to experimental data as much as possible, though they can be calculated from models in some cases.

To perform a less model-dependent method, we would like to use the standard distribution or its two-component form to obtain $T$ by fitting the experimental transverse momentum $\left(p_{\mathrm{T}}\right)$ or transverse mass $\left(m_{\mathrm{T}}\right)$ spectra of various particles. The standard distribution includes the Bose-Einstein, Fermi-Dirac, and Boltzmann distributions, in which the effective temperature parameter $T$ is the closest to that in the ideal gas model when comparing $T$ with those in other distributions. After the fitting, we hope to extract $T_{0}$ and $\beta_{\mathrm{T}}$ from the relation to average $p_{T}\left(\left\langle p_{T}\right\rangle\right)$ due to the Erlang distribution in the multisource thermal model [19-21] and $T_{\mathrm{i}}$ from the relation to root-mean-square $p_{T}\left(\sqrt{\left\langle p_{T}^{2}\right\rangle}\right)$ due to the color string percolation model [22-24]. Obviously, $\left\langle p_{T}\right\rangle$ and $\sqrt{\left\langle p_{T}^{2}\right\rangle}$ depend on the data themselves, though they can be calculated from the models.

In this work, the $p_{T}\left(m_{T}\right)$ spectra of positively and negatively charged pions $\left(\pi^{+}\right.$and $\left.\pi^{-}\right)$and positively and negatively charged kaons $\left(K^{+}\right.$and $\left.K^{-}\right)$, protons, and antiprotons $(p$ and $\bar{p}$ ) produced at mid-(pseudo)rapidity (mid- $y$ or mid$\eta)$ measured in central gold-gold ( $\mathrm{Au}-\mathrm{Au}$ ) collisions at the Alternating Gradient Synchrotron (AGS) by the E866 [25], E895 [26, 27], and E802 [28, 29] Collaborations and at the Relativistic Heavy Ion Collider (RHIC) by the STAR [3032] and PHENIX [33, 34] Collaborations, in central leadlead $(\mathrm{Pb}-\mathrm{Pb})$ collisions at the Super Proton Synchrotron (SPS) by the NA49 Collaboration [35-37] and at the Large Hadron Collider (LHC) by the ALICE Collaboration [38], as well as in inelastic (INEL) proton-proton $(p p)$ collisions at the SPS by the NA61/SHINE Collaboration [39, 40], at the RHIC by the PHENIX Collaboration [41], and at the LHC by the CMS Collaboration [42, 43], are studied. The (two-component) standard distribution is used to fit the data and to extract $T, T_{\mathrm{i}}, T_{0}$, and $\beta_{\mathrm{T}}$, as well as the excitation functions (energy dependences) of parameters.

The remainder of this paper is structured as follows. The formalism and method are shortly described in Section 2. Results and discussion are given in Section 3. In Section 4, we summarize our main observations and conclusions.

\section{Formalism and Method}

In high-energy collisions, the soft excitation and hard scattering processes are two main processes of particle productions. Most light flavor particles are produced in the soft excitation process and distributed in a narrow $p_{T}$ range which is less than $2 \sim 3 \mathrm{GeV} / c$ or a little more. Some light flavor particles are produced in the hard scattering process and distributed in a wide $p_{T}$ range. In collisions at not too high energies, the contribution of the hard scattering process can be neglected and the main contributor that produced particles is the soft excitation process. In collisions at a high energy, the contribution of the hard scattering process cannot be neglected, though the main contributor that produced particles is also the soft excitation process. It is expected that the contribution fraction of the hard scattering process increases with the increase of collision energy.

The contributions of soft excitation and hard scattering processes can be described by similar or different probability density functions. Generally, the hard scattering process does not contribute mainly to the temperature and flow velocity due to its small fraction in a narrow $p_{T}$ range. We can neglect the contribution of the hard scattering process if we study the spectra in a not too wide $p_{T}$ range. On the contribution of the soft excitation process, we have more than one functions to describe the $p_{T}$ spectra. These functions include, but are not limited to, the standard distribution [44], the Tsallis statistics [44-47], the Erlang distribution [19-21], the Schwinger mechanism [48-51], the blast-wave model with Boltzmann statistics [52, 53], the blast-wave model with Tsallis statistics [54-56], the Hagedorn thermal distribution [57], and their superposition with two- or three-component. These functions also describe partly the $p_{T}$ spectra of the hard scattering process in most cases.

In our opinion, in the case of fitting the data with acceptable representations, various distributions show similar behaviors which result in similar $\left\langle p_{T}\right\rangle\left(\sqrt{\left\langle p_{T}^{2}\right\rangle}\right)$ with different parameters. To be the closest to the temperature concept in the ideal gas model, we choose the standard distribution in which the chemical potential $\mu$ and spin property $S$ are included. That is, one has the probability density function in terms of $p_{T}$ to be [44]

$$
\begin{aligned}
f_{p_{T}}\left(p_{T}, T\right)= & \frac{1}{N} \frac{d N}{d p_{T}}=C p_{T} m_{T} \int_{y_{\min }}^{y_{\max }} \cosh y \\
& \times\left[\exp \left(\frac{m_{T} \cosh y-\mu}{T}\right)+S\right]^{-1} d y,
\end{aligned}
$$

where

$$
m_{T}=\sqrt{p_{T}^{2}+m_{0}^{2}}
$$

$m_{0}$ is the rest mass, $N$ denotes the particle number, $y_{\min }$ $\left(y_{\max }\right)$ is the minimum (maximum) value in the rapidity interval, $S=-1(+1)$ is for bosons (fermions), and $C$ is the normalization constant. Similarly, the probability density function in terms of $m_{T}$ is

$$
\begin{aligned}
f_{m_{T}}\left(m_{T}, T\right)= & \frac{1}{N} \frac{d N}{d m_{T}}=C m_{T}^{2} \int_{y_{\min }}^{y_{\max }} \cosh y \\
& \times\left[\exp \left(\frac{m_{T} \cosh y-\mu}{T}\right)+S\right]^{-1} d y .
\end{aligned}
$$

In some cases, the independent variable $m_{T}$ in Equation (3) is replaced by $m_{T}-m_{0}$ which starts at 0 . Both $m_{T}$ and $m_{T}-m_{0}$ show the same distribution shape. As 
probability density functions, the integrals of Equations (1) and (3) are naturally normalized to 1 , respectively.

The chemical potential $\mu$ in Equations (1) and (3) is particle dependent. For the particle type $i(i=\pi, K$, and $p$ in this work), its chemical potential $\mu_{i}$ is expressed by $[34,58,59]$

$$
\mu_{i}=-\frac{1}{2} T_{c h} \ln \left(k_{i}\right),
$$

where $k_{i}$ denotes the ratio of negative to positive particle numbers,

$$
T_{\mathrm{ch}}=\frac{T_{\lim }}{1+\exp \left[2.60-\ln \left(\sqrt{s_{N N}}\right) / 0.45\right]}
$$

is empirically the chemical freeze-out temperature in the statistical thermal model [15-18], $T_{\lim }=0.158 \mathrm{GeV}$ is the limiting or saturation temperature [3], and $\sqrt{s_{N N}}$ is the center-ofmass energy per nucleon pair in the units of $\mathrm{GeV}$.

Generally, one needs one or two standard distributions to fit the $p_{T}\left(m_{T}\right)$ spectra in a narrow range. In particular, if the resonance decays contribute a large fraction, a twocomponent distribution is indeed needed. Or, if the hard scattering process contributes a sizable fraction in the considered $p_{T}\left(m_{T}\right)$ range, a two-component distribution is also needed. In the case of using the two-component standard distribution in which the contributions from resonance decay are naturally included in the first component which covers the spectra in the low- $p_{T}$ region $(<0.2 \sim 0.3 \mathrm{GeV} / c)$, one has the probability density functions of $p_{T}$ and $m_{T}$ to be

$$
\begin{gathered}
f_{p_{T}}\left(p_{T}\right)=k f_{p_{T}}\left(p_{T}, T_{1}\right)+(1-k) f_{p_{T}}\left(p_{T}, T_{2}\right), \\
f_{m_{T}}\left(m_{T}\right)=k f_{m_{T}}\left(m_{T}, T_{1}\right)+(1-k) f_{m_{T}}\left(m_{T}, T_{2}\right),
\end{gathered}
$$

respectively, where $k$ denotes the contribution fraction of the first component, and $f_{p_{T}}\left(p_{T}, T_{1}\right)\left[f_{p_{T}}\left(p_{T}, T_{2}\right)\right]$ and $f_{m_{T}}\left(m_{T}, T_{1}\right)\left[f_{m_{T}}\left(m_{T}, T_{2}\right)\right]$ are given in Equations (1) and (3), respectively. The integrals of Equations (6) and (7) are also normalized to 1 , respectively. Correspondingly,

$$
T=k T_{1}+(1-k) T_{2}
$$

is averaged by weighting the two fractions. The temperature $T$ defined in Equation (8) reflects the common effective temperature of the two components in the case that the two components are assumed to stay in equilibrium.

According to the Hagedorn model [57], one may also use the usual step function $\theta(x)$ to superpose the two standard distributions, where $\theta(x)=0$ if $x<0$ and $\theta(x)=1$ if $x \geq 0$. Thus, we have new probability density functions of $p_{T}$ and $m_{T}$ to be

$$
f_{p_{T}}\left(p_{T}\right)=A_{1} \theta\left(p_{1}-p_{T}\right) f_{p_{T}}\left(p_{T}, T_{1}\right)+A_{2} \theta\left(p_{T}-p_{1}\right) f_{p_{T}}\left(p_{T}, T_{2}\right),
$$

$$
\begin{aligned}
f_{m_{T}}\left(m_{T}\right)= & A_{1} \theta\left(m_{1}-m_{T}\right) f_{m_{T}}\left(m_{T}, T_{1}\right) \\
& +A_{2} \theta\left(m_{T}-m_{1}\right) f_{m_{T}}\left(m_{T}, T_{2}\right),
\end{aligned}
$$

respectively, where $A_{1}$ and $A_{2}$ are constants which result in the two components to be equal to each other at $p_{T}=p_{1}$ and $m_{T}=m_{1}$. The integrals of Equations (9) and (10) should be normalized to 1 , respectively, due to the fact that they are probability density functions. The contribution fractions of the first component in Equations (9) and (10) are

$$
\begin{aligned}
k= & \int_{0}^{p_{1}} A_{1} f_{p_{T}}\left(p_{T}, T_{1}\right) d p_{T} \\
& =1-\int_{p_{1}}^{p_{T} \max } A_{2} f_{p_{T}}\left(p_{T}, T_{2}\right) d p_{T}, \\
k & =\int_{m_{0}}^{m_{1}} A_{1} f_{m_{T}}\left(m_{T}, T_{1}\right) d m_{T} \\
& =1-\int_{m_{1}}^{m_{T} \max } A_{2} f_{m_{T}}\left(m_{T}, T_{2}\right) d m_{T},
\end{aligned}
$$

respectively, where $p_{T \text { max }}$ and $m_{T \text { max }}$ denote the maximum $p_{T}$ and $m_{T}$, respectively. Equation (8) is also suitable for the superposition in terms of the Hagedorn model [57].

The two superpositions show respective advantages and disadvantages. The first superposition can fit the data by a smooth curve. However, there are correlations in determining $T_{1}$ and $T_{2}$. The second superposition can determine $T_{1}$ and $T_{2}$ without correlations. However, the curves are possibly not smooth at $p_{1}$ or $m_{1}$. In the case of obtaining $\left\langle p_{T}\right\rangle$ and $\sqrt{\left\langle p_{T}^{2}\right\rangle}$, it does not matter which superposition is used, though the two $T$ are slightly different. In this work, we use the first superposition to obtain smooth curves. One has

$$
\begin{gathered}
\left\langle p_{T}\right\rangle=\int_{0}^{p_{T} \max } p_{T} f_{p_{T}}\left(p_{T}\right) d p_{T}, \\
\sqrt{\left\langle p_{T}^{2}\right\rangle}=\sqrt{\int_{0}^{p_{T} \max } p_{T}^{2} f_{p_{T}}\left(p_{T}\right) d p_{T},}
\end{gathered}
$$

due to

$$
\int_{0}^{p_{T} \max } f_{p_{T}}\left(p_{T}\right) d p_{T}=1
$$

Based on the $m_{T}$ spectrum, we may use the same parameters to obtain $\left\langle p_{T}\right\rangle$ and $\sqrt{\left\langle p_{T}^{2}\right\rangle}$ from the related formula of $p_{T}$ distribution.

It should be noted that, since we aim to extract the parameters in a less model-dependent way, we shall obtain $\left\langle p_{T}\right\rangle$ and $\sqrt{\left\langle p_{T}^{2}\right\rangle}$ from the combination of data points and fit function in this paper. In fact, we may divide $p_{T}\left(m_{T}\right)$ spectrum into two or three regions according to the measured and unmeasured $p_{T}\left(m_{T}\right)$ ranges. To obtain $\left\langle p_{T}\right\rangle$ and $\sqrt{\left\langle p_{T}^{2}\right\rangle}$, we may use the data points in the measured $p_{T}\left(m_{T}\right.$ 
) range and only use the fit function to extrapolate to the unmeasured $p_{T}\left(m_{T}\right)$ range.

In each nucleon-nucleon collision in AA and pp collisions, the projectile and target participant sources contribute equally to $\left\langle p_{T}\right\rangle$. In the framework of the multisource thermal model [19-21], each projectile and target source contribute a fraction of $1 / 2$ to $\left\langle p_{T}\right\rangle$, i.e., $\left\langle p_{T}\right\rangle / 2$ which is contributed together by the thermal motion and flow effect. Let $k_{0}$ $\left(1-k_{0}\right)$ denote the contribution fraction of thermal motion (flow effect); we define empirically

$$
\begin{aligned}
& T_{0} \equiv \frac{k_{0}\left\langle p_{T}\right\rangle}{2}, \\
& \beta_{T} \equiv \frac{\left(1-k_{0}\right)\left\langle p_{T}\right\rangle}{2 m_{0} \bar{\gamma}},
\end{aligned}
$$

where $\bar{\gamma}$ is the mean Lorentz factor of the considered particles and

$$
k_{0}=0.30-0.01 \ln \left(\sqrt{s_{N N}}\right)
$$

is a parameterized representation in this paper due to our comparison with the results $[12,13]$ from the blast-wave model [52-56]. In Equation (18), $\sqrt{s_{N N}}$ is in the units of $\mathrm{GeV}$ as that in Equation (5).

In a recent work [60], it is shown that the effective temperature is proportional to $\left\langle p_{T}\right\rangle$ and the kinetic freeze-out temperature is proportional to the effective temperature, though the effective temperature used in Ref. [60] is different from this paper. This confirms the relation of $T_{0} \propto\left\langle p_{T}\right\rangle$ (Equation (16)) used in this paper. Considering each projectile and target source contributing $\left\langle p_{T}\right\rangle / 2$ [19-21], we have concretely $T_{0} \propto\left\langle p_{T}\right\rangle / 2$. The remainder in $\left\langle p_{T}\right\rangle / 2$ is naturally contributed by the transverse flow. This confirms Equations (16) and (17) to be justified, though $k_{0}$ is an empirical representation.

To continue this work, we need some assumptions and a coordinate system. In the source rest frame, the particles are assumed to emit isotropically. Meanwhile, the interactions among various sources are neglected, which affects slightly the $p_{T}\left(m_{T}\right)$ spectra, through which affects largely anisotropic flows [61]. A right-handed coordinate system $O-x y z$ is established in the source rest frame, where the $\mathrm{O} z$ axis is along the beam direction, the $x O y$ plane is the transverse plane, and the $x \mathrm{Oz}$ plane is the reaction plane.

We can obtain $\bar{\gamma}$ by a Monte Carlo (MC) method. Let $R_{1,2,3}$ denote a random number distributed evenly in $[0,1]$; each concrete $p_{T}$ satisfies

$$
\int_{0}^{p_{T}} f_{p_{T}}\left(p_{T}^{\prime}, T\right) d p_{T}^{\prime}<R_{1}<\int_{0}^{p_{T}+\delta p_{T}} f_{p_{T}}\left(p_{T}^{\prime}, T\right) d p_{T}^{\prime}
$$

where $\delta p_{T}$ denotes a small shift relative to $p_{T}$. Each concrete emission angle $\theta$ satisfies

$$
\theta=2 \arcsin \sqrt{R_{2}}
$$

due to the fact that $\theta$ obeys the probability density function $f_{\theta}(\theta)=(1 / 2) \sin \theta$ in $[0, \pi]$ in the case of isotropic assumption in the source rest frame. The solution of the equation $\int_{0}^{\theta} f_{\theta}{ }^{\prime}\left(\theta^{\prime}\right) d \theta^{\prime}=R_{2}$ is Equation (20). We give up to use rapidity due to the fact that it is unnecessary here. Each concrete momentum $p$, energy $E$, and Lorentz factor $\gamma$ can be obtained by

$$
\begin{aligned}
& p=p_{T} \csc \theta, \\
& E=\sqrt{p^{2}+m_{0}^{2}}, \\
& \gamma=\frac{E}{m_{0}},
\end{aligned}
$$

respectively. After multiple repeating calculations due to the MC method, we have

$$
\bar{\gamma}=\frac{\bar{E}}{m_{0}},
$$

where $\bar{E}$ denotes the mean $E$ for a given type of particle.

In addition, each concrete azimuthal angle $\phi$ satisfies

$$
\phi=2 \pi R_{3}
$$

due to the fact that $\phi$ obeys the probability density function $f_{\phi}(\phi)=1 /(2 \pi)$ in $[0,2 \pi]$ in the case of isotropic assumption in the source rest frame. The solution of the equation $\int_{0}^{\phi} f_{\phi}^{\prime}\left(\phi^{\prime}\right) d \phi^{\prime}=R_{3}$ is Equation (25). Each concrete momentum components $p_{x}, p_{y}$, and $p_{z}$ can be obtained by

$$
\begin{aligned}
& p_{x}=p_{T} \cos \phi, \\
& p_{y}=p_{T} \sin \phi, \\
& p_{z}=p_{T} \cot \theta=p \cos \theta,
\end{aligned}
$$

respectively. By using the components and $E$, $p$, and $\theta$, we can obtain other quantities such as (pseudo)rapidity and event structure [61] which are beyond the focus of this work and will not be studied anymore.

According to the color string percolation model [22-24], one has

$$
T_{i} \equiv \sqrt{\frac{\left\langle p_{T}^{2}\right\rangle}{2}}
$$

Meanwhile, we have the relation between the three components $p_{x}, p_{y}$, and $p_{z}$ of the momentum $p$ to be

$$
\sqrt{\left\langle p_{x}^{2}\right\rangle}=\sqrt{\left\langle p_{y}^{2}\right\rangle}=\sqrt{\left\langle p_{z}^{2}\right\rangle}=\sqrt{\frac{\left\langle p_{T}^{2}\right\rangle}{2}}
$$


in which the root-mean-square components $\sqrt{\left\langle p_{x}^{2}\right\rangle}, \sqrt{\left\langle p_{y}^{2}\right\rangle}$, and $\sqrt{\left\langle p_{z}^{2}\right\rangle}$ are used. Naturally, $T_{i}$ can be given by one of the root-mean-square components.

We would like to point out that the above isotropic assumption is only performed in the source rest frame. It is expected that many sources are formed in high-energy collisions according to the multisource model [19-21]. These sources distribute at different rapidities in the rapidity space, which appear at the effect of longitudinal flow. The twocomponent $p_{T}$ and $m_{T}$ spectra render that these sources stay in two different excitation states or have two different decay mechanisms. The interactions among these sources also affect anisotropic flows in transverse plane [61].

\section{Results and Discussion}

Figures $1(\mathrm{a})-1(\mathrm{q})$ show the $p_{T}\left(m_{T}-m_{0}\right)$ spectra, $\left(1 / 2 \pi p_{T}\right)$ - $d^{2} N / d y d p_{T}\left[\left(1 / 2 \pi m_{T}\right) \cdot d^{2} N / d y d m_{T}\right]$, of $\pi^{+}, \pi^{-}, K^{+}, K^{-}, p$ , and $\bar{p}$ produced at mid- $y$ or mid- $\eta$ in central Au-Au collisions at different $\sqrt{s_{N N}}$, where the particle types, $y$ or $\eta$ intervals, centrality classes, and collision energies are marked in the panels. The closed and open symbols represent, respectively, the experimental data of positively and negatively charged particles measured by the E866 [25], E895 [26, 27], E802 [28, 29], STAR [30-32], and PHENIX [33, 34] Collaborations marked in the panels, where in Figures 1(a)-1(d), the data for $\pi^{ \pm}$and $K^{+}$are taken from the E866 Collaboration [25] and the data for $p$ are taken from the E895 Collaboration $[26,27]$. The solid and dashed curves are our results fitted by Equation (6) or (7) for positively and negatively charged particles, respectively. The values of free parameters $\left(T_{1}, T_{2}\right.$ if available, and $k$ ), derived parameter $(T)$, normalization constant $\left(N_{0}\right), \chi^{2}$, and degree-of-freedom (dof) are listed in Table 1 . The dot-dashed curves are our results fitted by using the single component function with the weighted average parameter $\langle T\rangle$ which will be discussed later. The dotted curves and asterisks in Figures 1(a)-1(f) represent the MC results for $K^{+}$with high ( $10^{6}$ particles) and low ( $10^{4}$ particles) statistics, respectively, which will be discussed at the end of this section. In the fitting process for the solid and dashed curves, the least squares method is used to determine the best parameter values. The experimental global uncertainties used in the calculation of $\chi^{2}$ are taken to be the root sum square of statistical uncertainties and point-by-point systematic uncertainties. The best parameters are determined due to the limitation of the minimum $\chi^{2}$. The global uncertainties of parameters are obtained using the method of statistical simulation [62]. We note that $\chi^{2}$ per dof $\left(\chi^{2} /\right.$ dof $)$ in a few cases is larger than 10, which renders that the fit is not too good. One can see that the (two-component) standard distribution fits approximately the $p_{T}\left(m_{T}-m_{0}\right)$ spectra of $\pi^{ \pm}, K^{ \pm}, p$, and $\bar{p}$ measured at mid- $y$ or mid- $\eta$ in central Au-Au collisions over an energy range from 2.7 to $200 \mathrm{GeV}$ in most cases.

Figure 2 is similar to Figure 1, but it is showing the spectra of various particles produced at mid- $y$ in central $\mathrm{Pb}-\mathrm{Pb}$ collisions at high $\sqrt{s_{N N}}$, where the factor $(1 / 2 \pi)$ on the vertical axis is removed in some cases and $N_{\text {evt }}$ if available denotes the particle number which can be removed. The symbols represent the experimental data measured by the NA49 [35-37] and ALICE [38] Collaborations. The values of various parameters, $\chi^{2}$, and dof for fitting the solid and dashed curves are listed in Table 2. Figure 3 is similar to Figures 1 and 2, but it showing the spectra of various particles produced at mid- $y$ or mid- $\eta$ in INEL $p p$ collisions at high center-of-mass energies $\sqrt{s}$, where the factor $\left(1 / 2 \pi p_{T}\right)$ on the vertical axis is removed and the invariant cross-section $E d^{3} \sigma / d p^{3}$ is used in some cases. The symbols represent the experimental data measured by the NA61/SHINE [39, 40], PHENIX [41], and CMS $[42,43]$ Collaborations. The values of various parameters, $\chi^{2}$, and dof for fitting the solid and dashed curves are listed in Table 3, where the cross-section $\sigma_{0}$ is used as the normalization constant if the spectrum is $E d^{3} \sigma / d p^{3}$. One can see that the (two-component) standard distribution fits approximately the $p_{T}\left(m_{T}-m_{0}\right)$ spectra of $\pi^{ \pm}, K^{ \pm}, p$, and $\bar{p}$ measured at mid- $y$ or mid- $\eta$ in central Pb-Pb and INEL $p p$ collisions over an energy range from 6.3 to $13000 \mathrm{GeV}$ in most cases.

To study the dependence of the main parameter $T$ on collision energy $\sqrt{s_{N N}}$ or $\sqrt{s}$, the excitation functions of $T$ for central $\mathrm{Au}-\mathrm{Au}(\mathrm{Pb}-\mathrm{Pb})$ collisions and INEL $p p$ collisions are shown in Figures 4(a) and 4(b), respectively. The results obtained from the spectra of $\pi^{+}, \pi^{-}, K^{+}, K^{-}, p$, and $\bar{p}$ are displayed by different symbols marked in the panels. The asterisks represent $\langle T\rangle$ which is the average $T$ by weighting different masses and yields of the six particles. In the case where one of the six particles is absent, $\langle T\rangle$ is not available. One can see that $T$ and $\langle T\rangle$ increase with the increase of $\ln$ $\left(\sqrt{s_{N N}}\right)[\ln (\sqrt{s})]$. Meanwhile, $T$ increases with the increase of particle mass.

To better determine the kinetic freeze-out information, we now fit simultaneously the spectra of $\pi^{+}, \pi^{-}, K^{+}, K^{-}, p$, and $\bar{p}$ in different $p_{T}$ ranges using the same set of parameters. In Figures 1-3, the dot-dashed curves are the results using the weighted average $\langle T\rangle$ which is energy dependent, though in fact we may use the other $T$ to obtain a little better result in some cases. In the refit, the normalization constant $N_{0}$ for different spectra is adjustable to fit the suitable $p_{T}$ range. In despite of mass-dependent (two-)temperature in the nonsimultaneous fit, the simultaneous fit is done using the same and only set of $\langle T\rangle$ for the six species of particles at each energy. That is to say that we use one component function to fit the spectra of the six species of particles at each energy. And the temperature is the same for each particle, and the normalization factor is adjusted. It seems that our treatment is not a fair comparison of the nonsimultaneous fit and simultaneous fit, where the nonsimultaneous fit uses the two-component in some cases. However, the simultaneous fit seeks for the same temperature which does not allow the two-component for some particles due to other particles corresponding to one component. In our opinion, our comparison is fair for the nonsimultaneous fit and the simultaneous fit. One can see that the same set of $\langle T\rangle$ can fit only a part of the $p_{T}$ range in some cases. The fit results using the same set of parameters are not ideal. These results do not support the single scenario for kinetic freeze-out. 

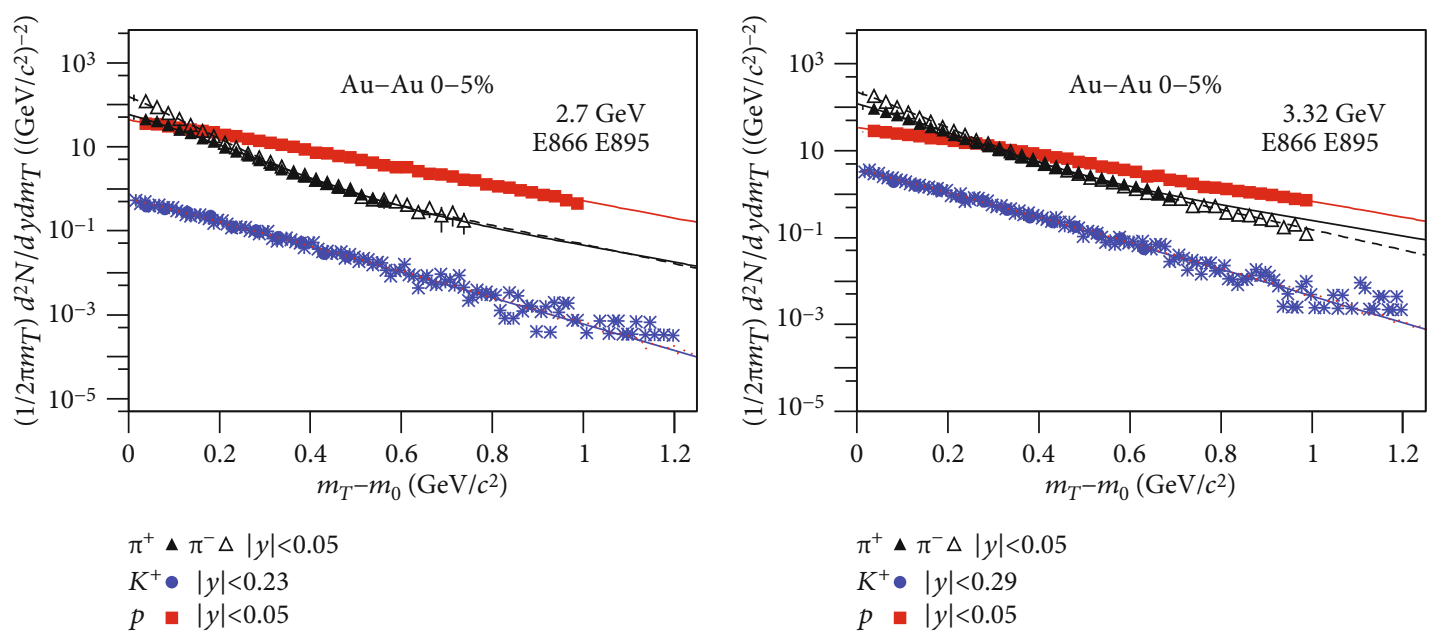

(a)

(b)
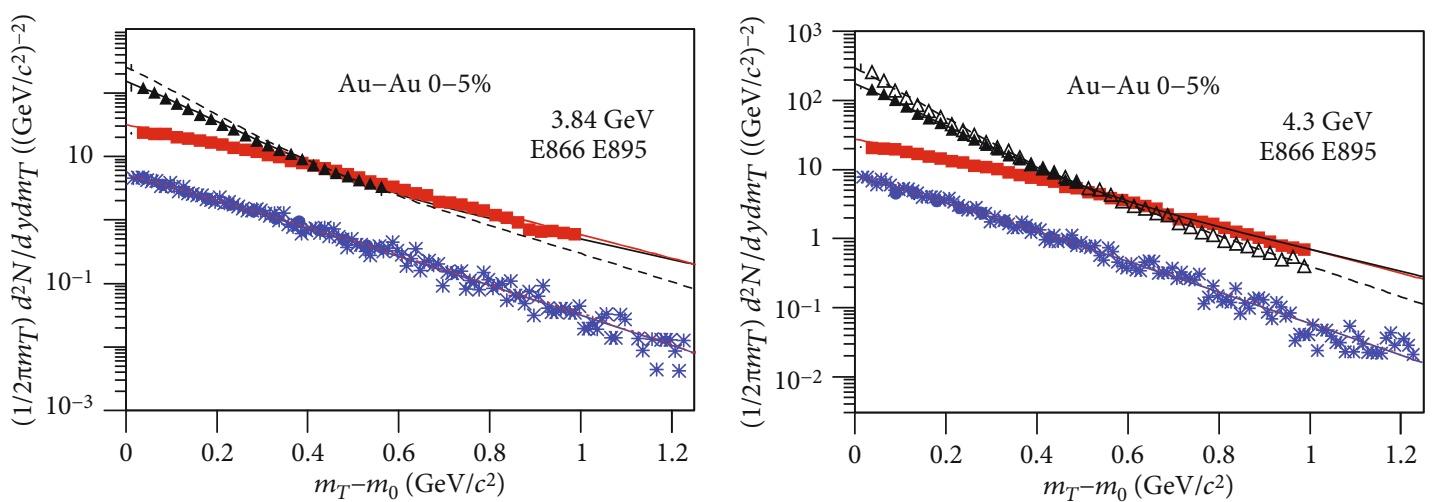

$\pi^{+} \Delta \pi^{-} \Delta|y|<0.05$

$K^{+} \bullet|y|<0.34$

$\pi^{+} \Delta \pi^{-} \Delta|y|<0.05$

$K^{+} \bullet|y|<0.37$

$p=|y|<0.05$

(c)
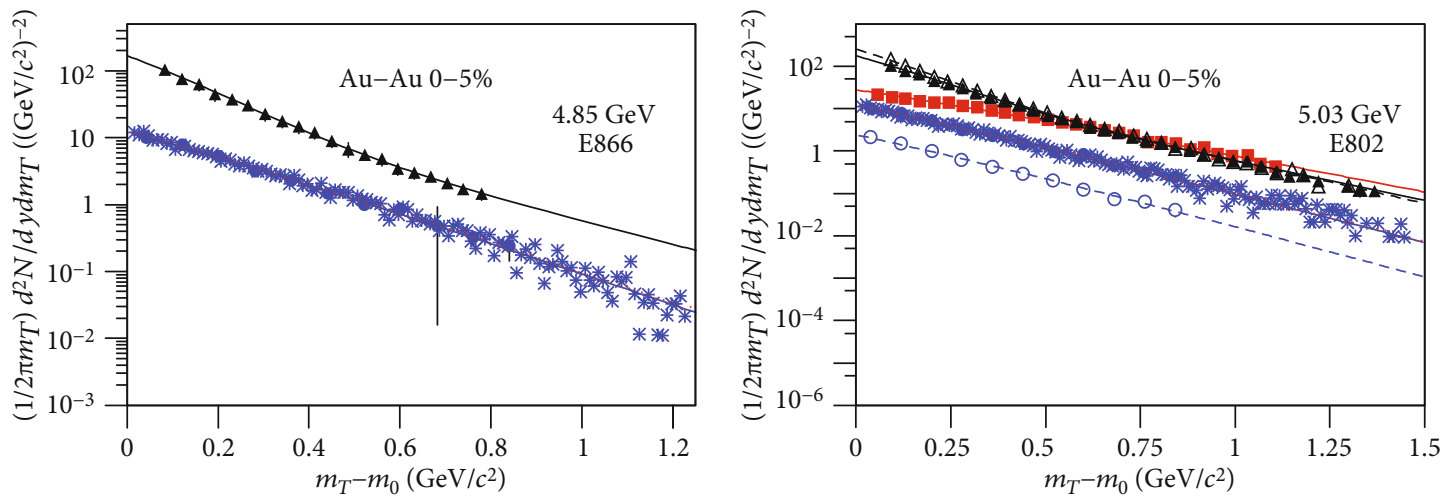

$\pi^{+} \boldsymbol{\Delta}|y|<0.2$

$\pi^{+} \triangle \pi^{-} \Delta 0<y<0.4$

$K^{+} \bullet K^{-} \circ|y|<0.1$

$p=\quad 0<y<0.2$

(e)

(f)

FIgURe 1: Continued. 


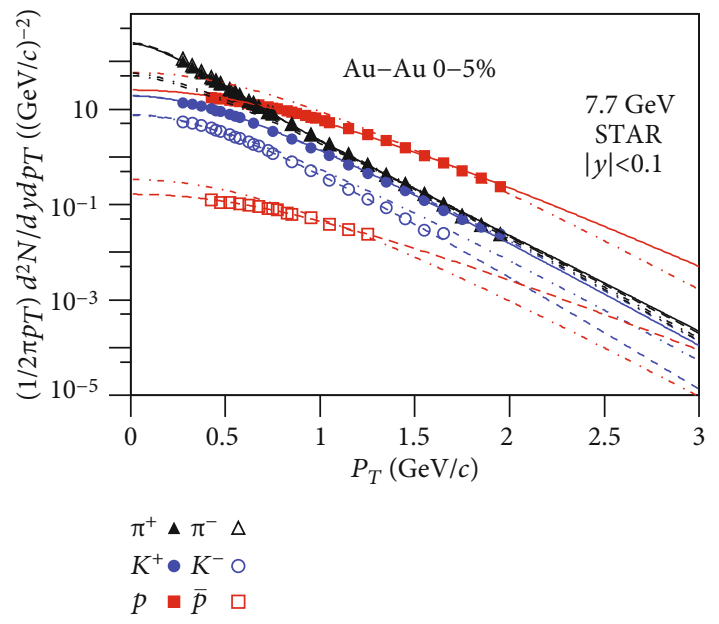

(g)

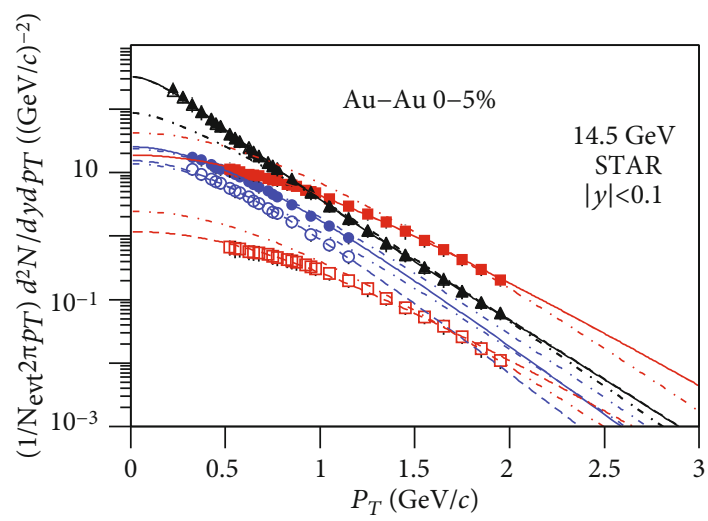

$\pi^{+} \Delta \pi^{-} \Delta$

$K^{+} \bullet K^{-}$。

$p \backsim \bar{p} \square$

(i)

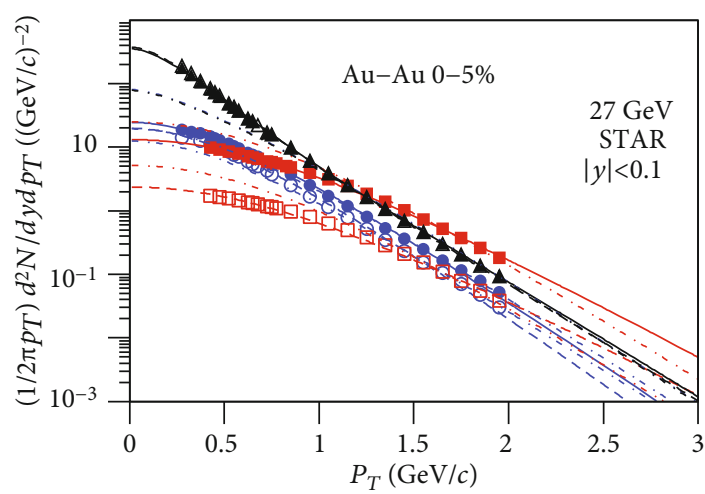

$\pi^{+} \Delta \pi^{-} \Delta$

$K^{+} \bullet K^{-} \circ$

$p=\bar{p} \square$

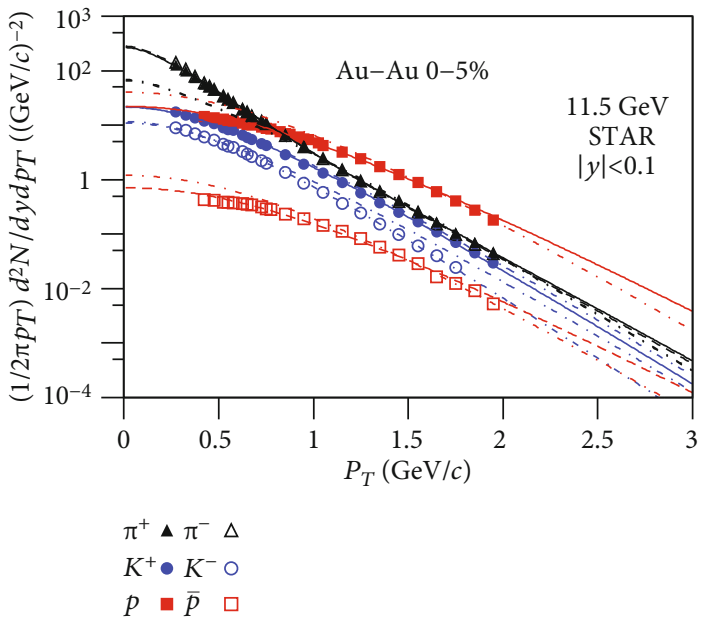

(h)

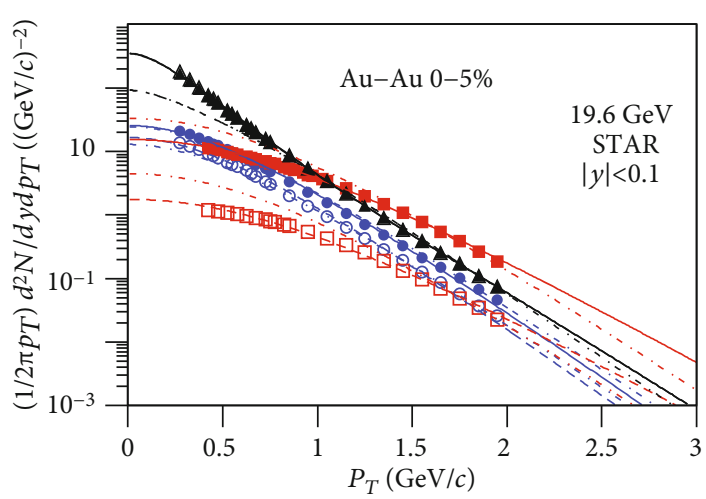

$\pi^{+} \Delta \pi^{-} \Delta$
$K^{+} \bullet K^{-} \circ$
$p=\bar{p}$

(j)

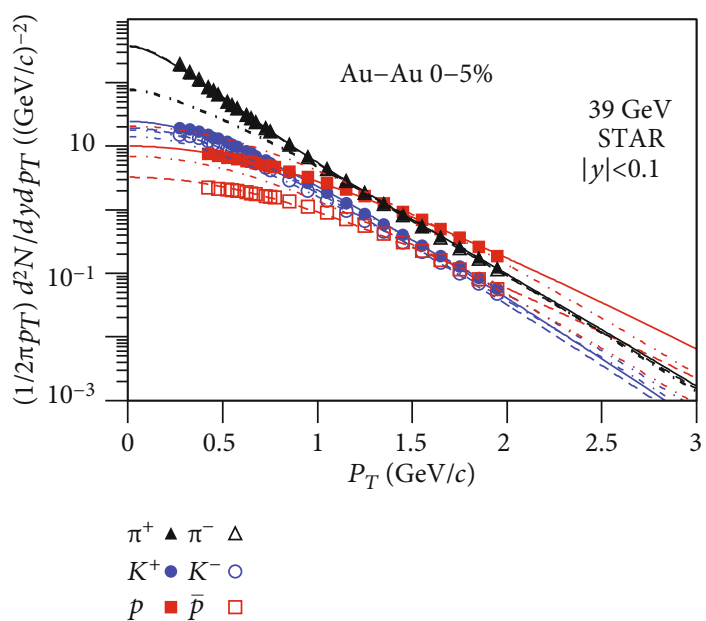

(l)

Figure 1: Continued. 


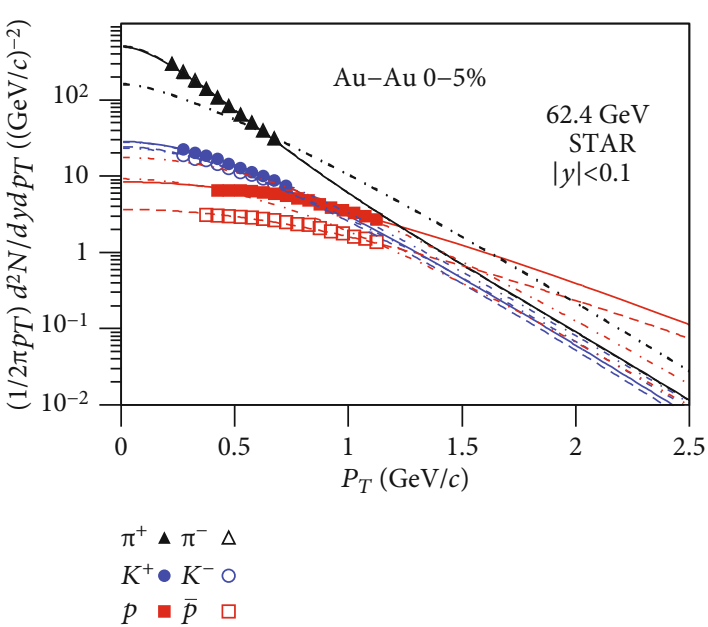

(m)

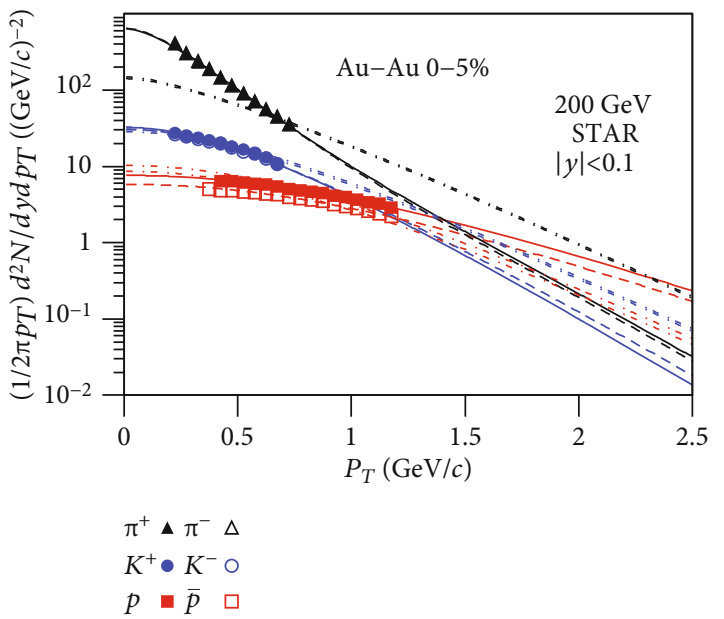

(o)

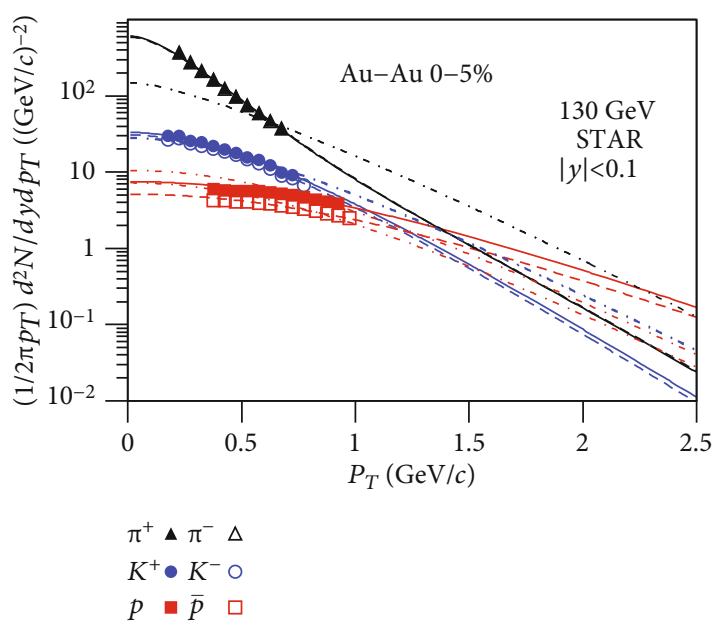

(n)

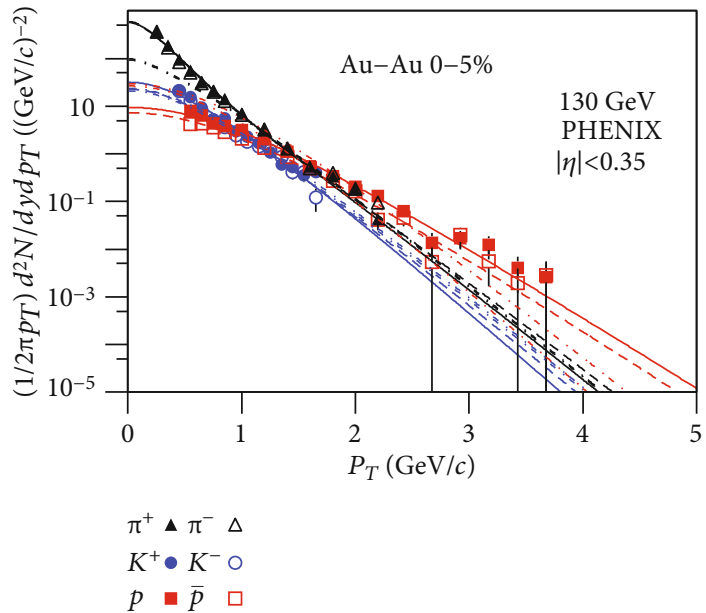

(p)

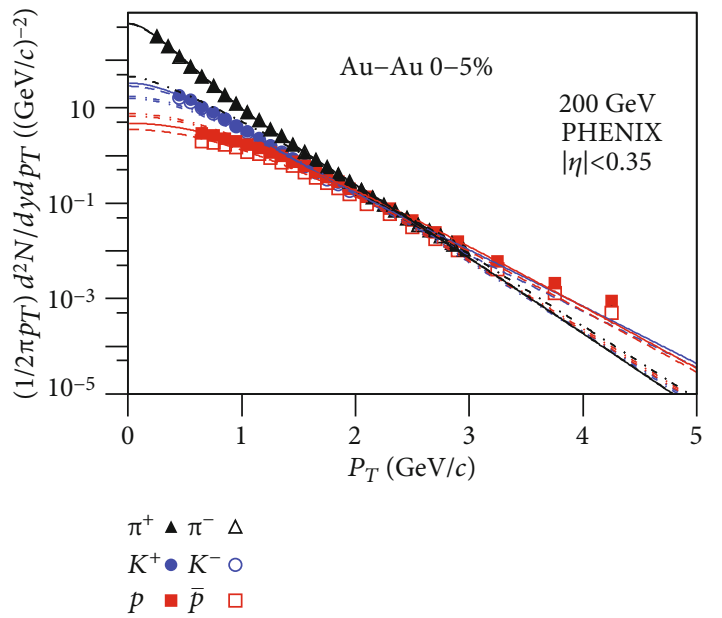

(q)

Figure 1: The $p_{T}\left(m_{T}-m_{0}\right)$ spectra, $\left(1 / 2 \pi p_{T}\right) \cdot d^{2} N / d y d p_{T}\left[\left(1 / 2 \pi m_{T}\right) \cdot d^{2} N / d y d m_{T}\right]$, of $\pi^{+}, \pi^{-}, K^{+}, K^{-}, p$, and $\bar{p}$ produced at mid- $y$ or mid- $\eta$ in central Au-Au collisions at high $\sqrt{s_{N N}}$. The closed and open symbols represent, respectively, the experimental data of positively and negatively charged particles measured by (a-e) the E866 [25] and E895 [26, 27], (f) the E802 [28, 29], (g-o) the STAR [30-32], and (p-q) the PHENIX $[33,34]$. Collaborations marked in the panels which appear mostly $(g-q)$, where in (a)-(d), the data for $\pi^{ \pm}$and $K^{+}$are taken from the E866 Collaboration [25] and the data for $p$ are taken from the E895 Collaboration [26, 27]. The solid and dashed curves are our results fitted by Equation (6) or (7) for positively and negatively charged particles, respectively. The dot-dashed curves are our results fitted by using the weighted average parameter $\langle T\rangle$. The dotted curves and asterisks in (a)-(f) represent the MC results for $K^{+}$with high (10 $0^{6}$ particles) and low $\left(10^{4}\right.$ particles) statistics, respectively. 


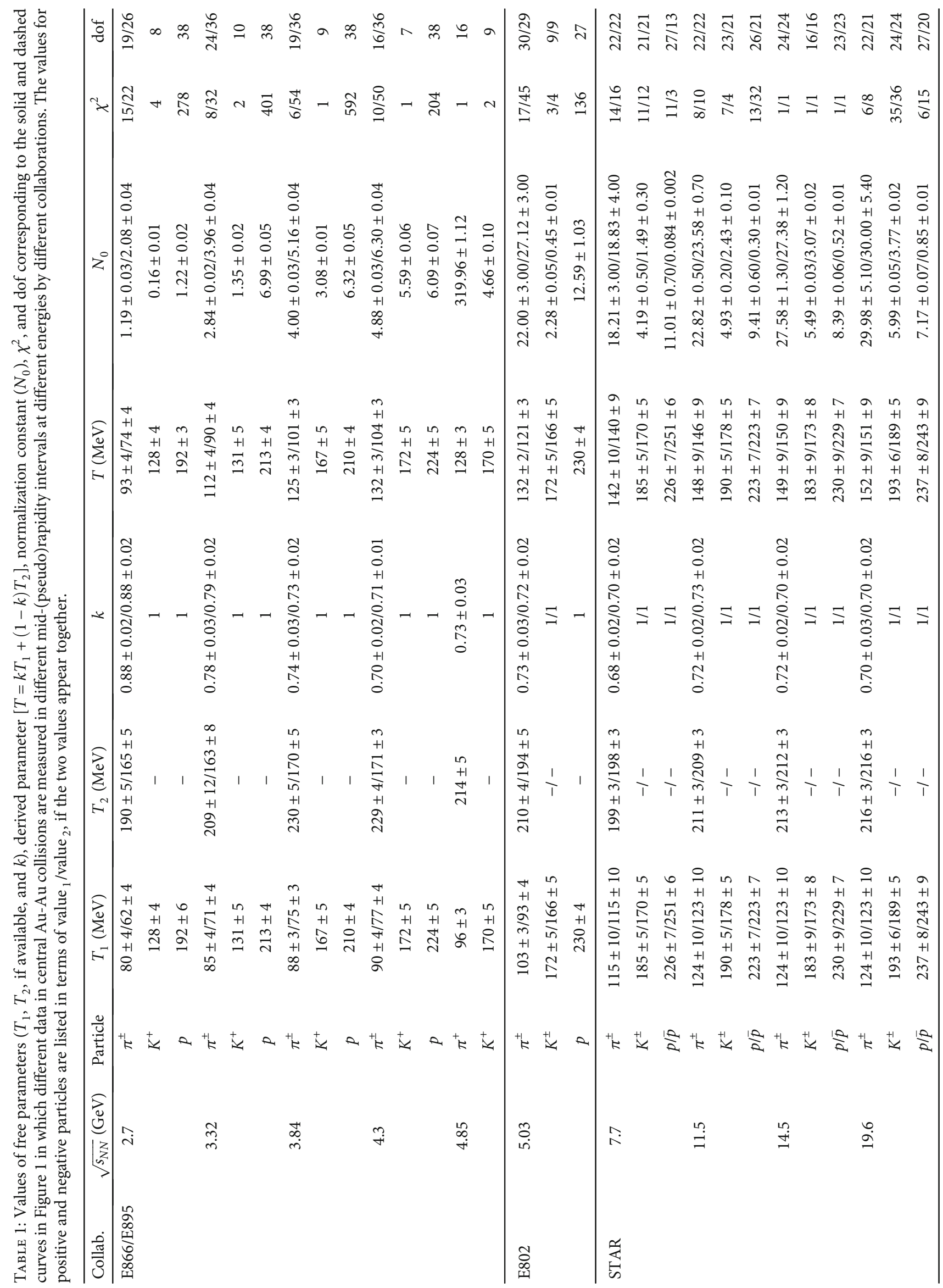




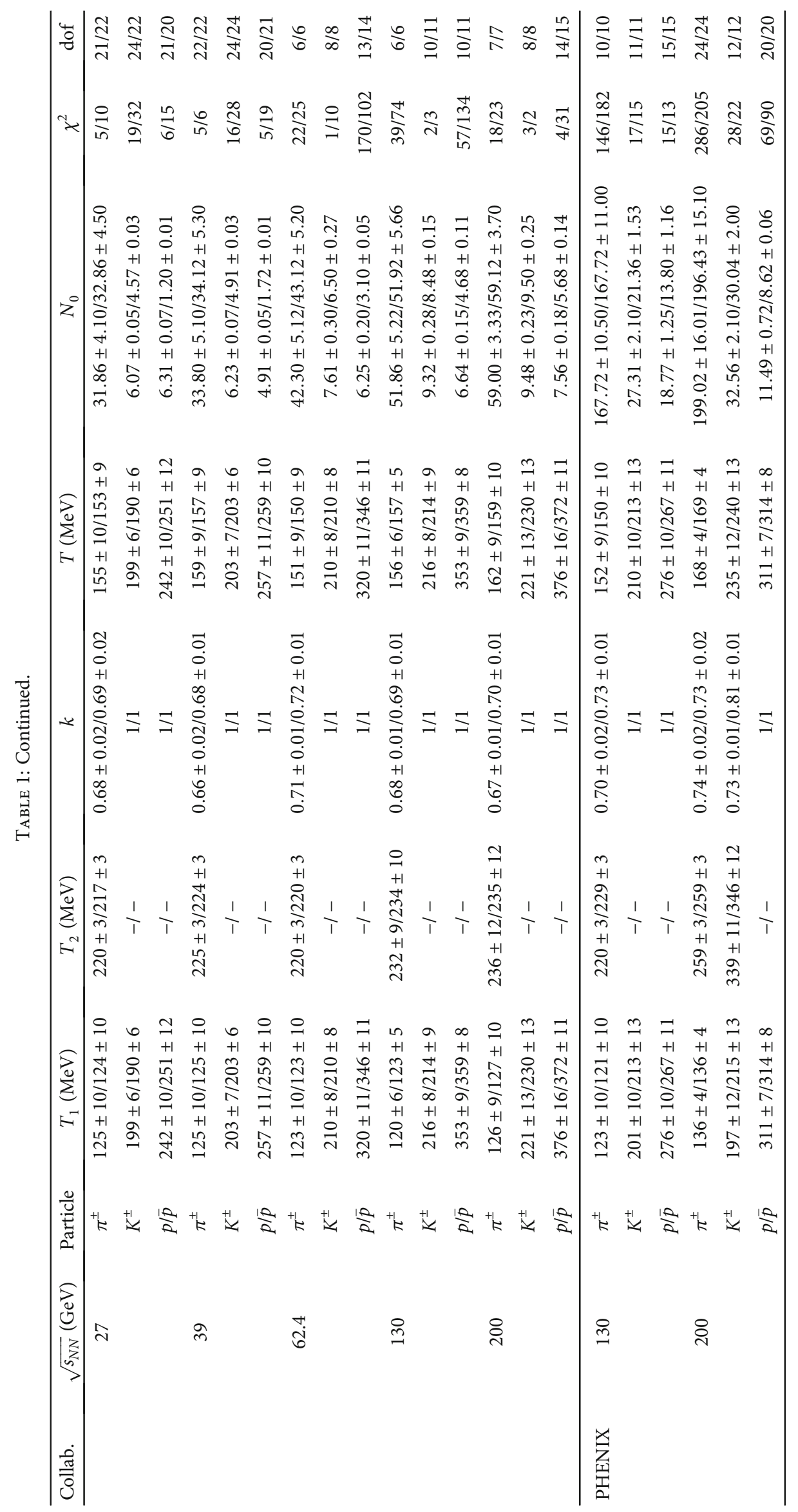




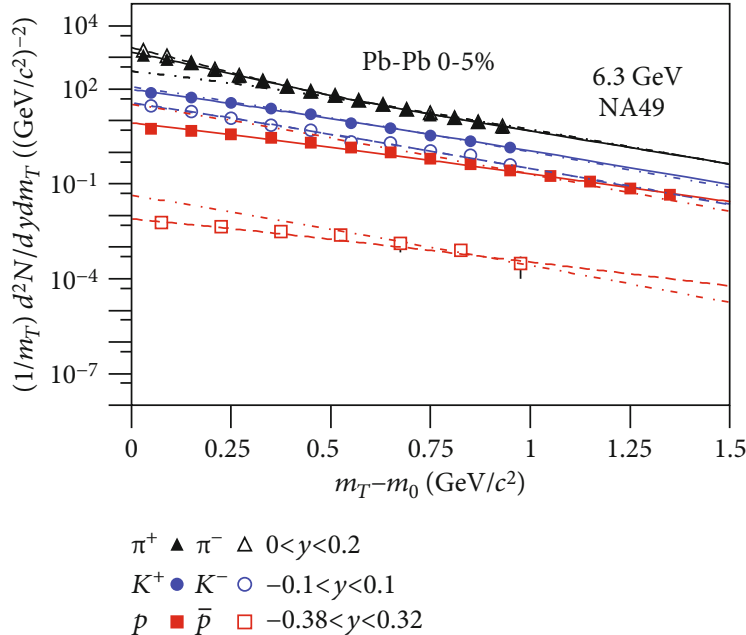

(a)

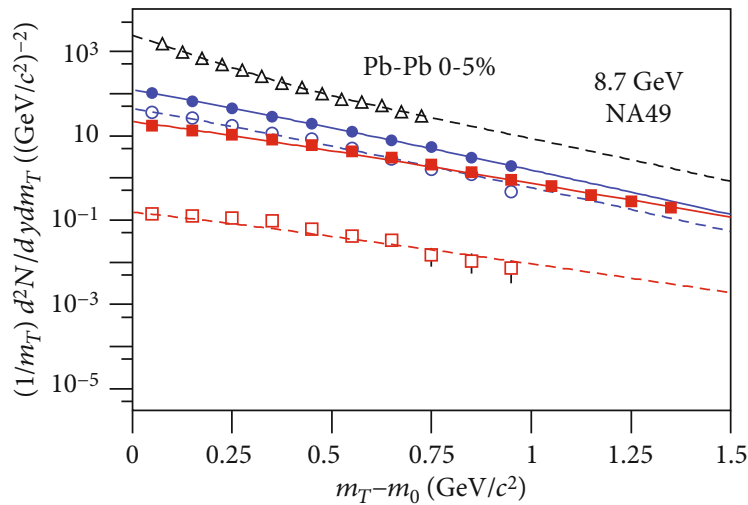

$$
\begin{aligned}
& \pi^{-} \Delta \quad 0<y<0.2 \\
& K^{+} \bullet K^{-} \circ-0.1<y<0.1 \\
& p=\bar{p} \quad \square-0.32<y<0.08
\end{aligned}
$$

(c)

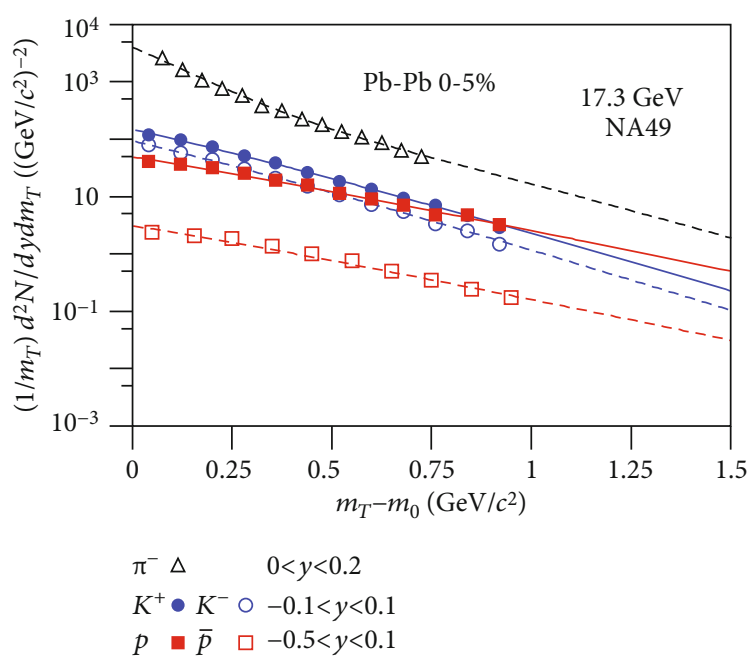

(e)

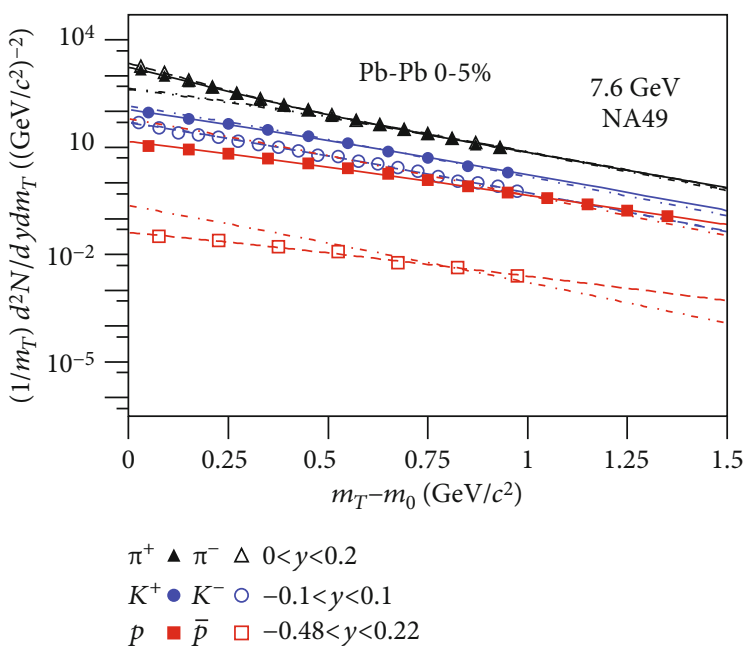

(b)

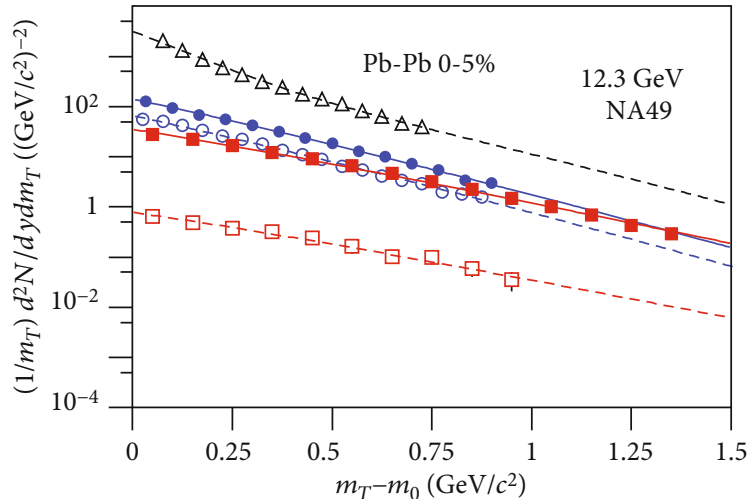

$$
\begin{array}{ll}
\pi^{-} \Delta & 0<y<0.2 \\
K^{+} \bullet K^{-} \circ-0.1<y<0.1 \\
p \quad \bar{p} \quad \square-0.37<y<0.03
\end{array}
$$

(d)

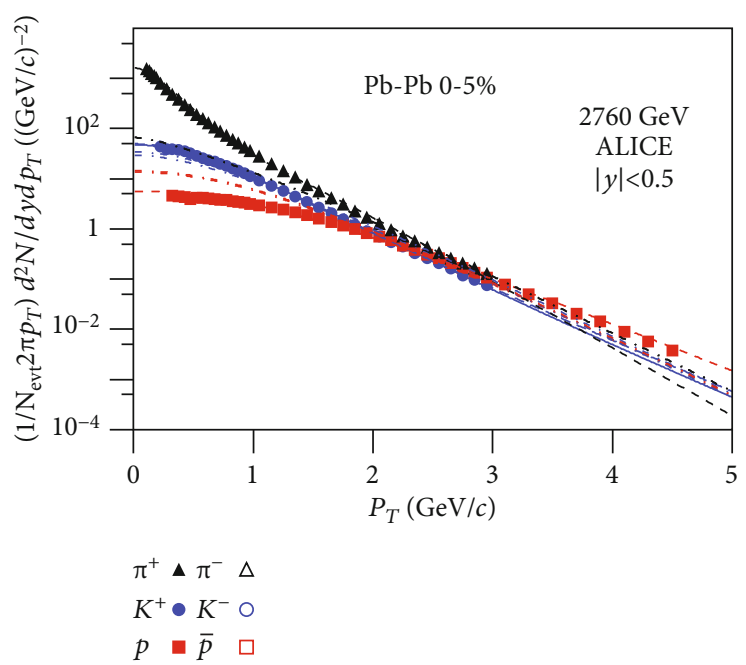

(f)

FIGURE 2: Same as in Figure 1 but showing the spectra of various particles produced at mid- $y$ in central Pb-Pb collisions at high $\sqrt{s_{N N}}$, where the factor $(1 / 2 \pi)$ on the vertical axis is removed in some cases and $N_{\text {evt }}$ if available denotes the particle number which can be removed. The symbols represent the experimental data measured by (a-e) the NA49 [35-37] and (f) the ALICE [38] Collaborations. 


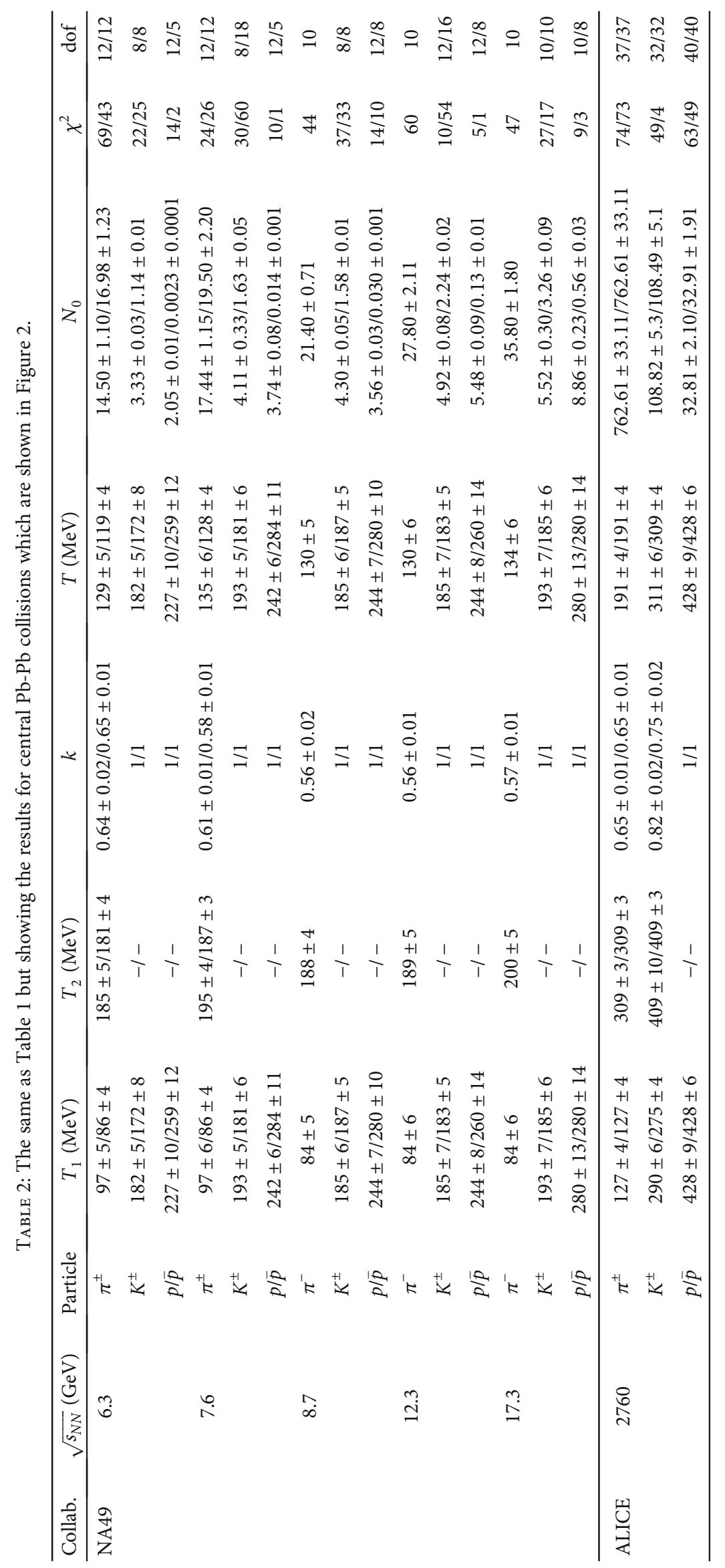



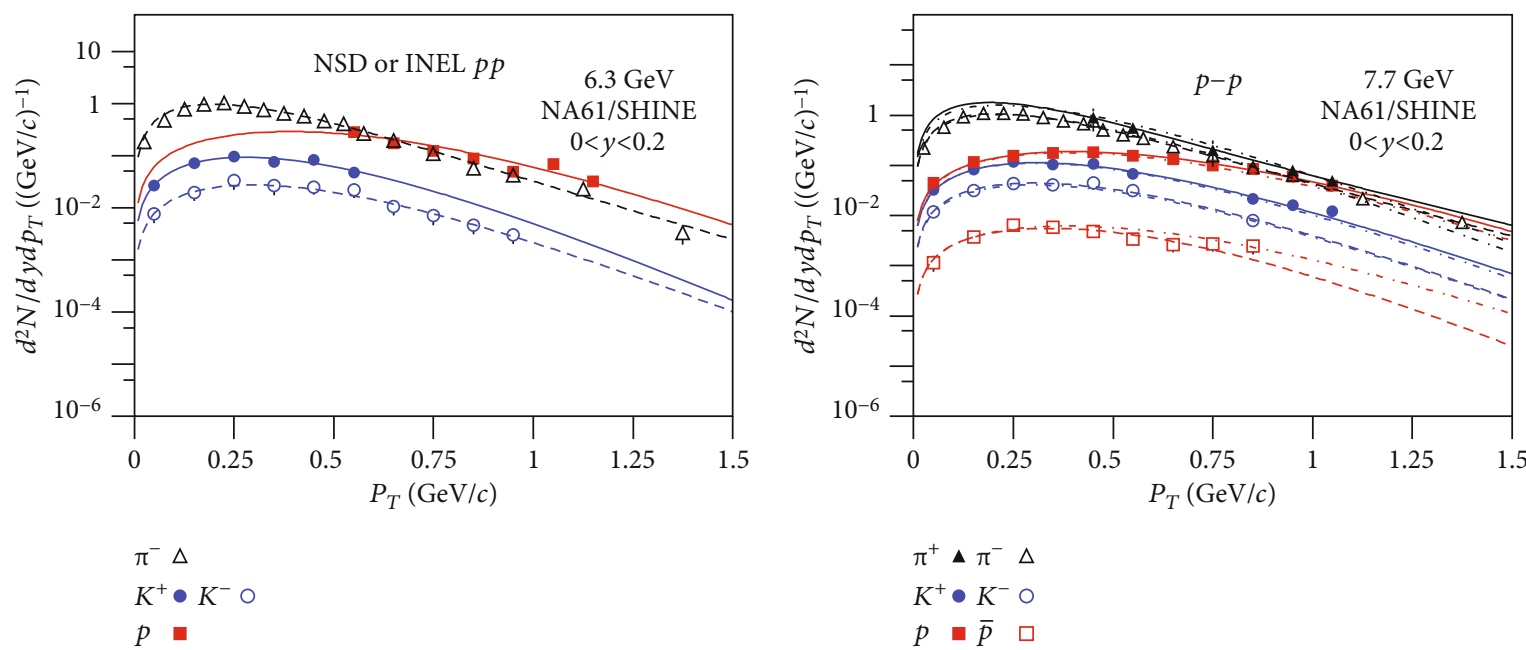

(a)

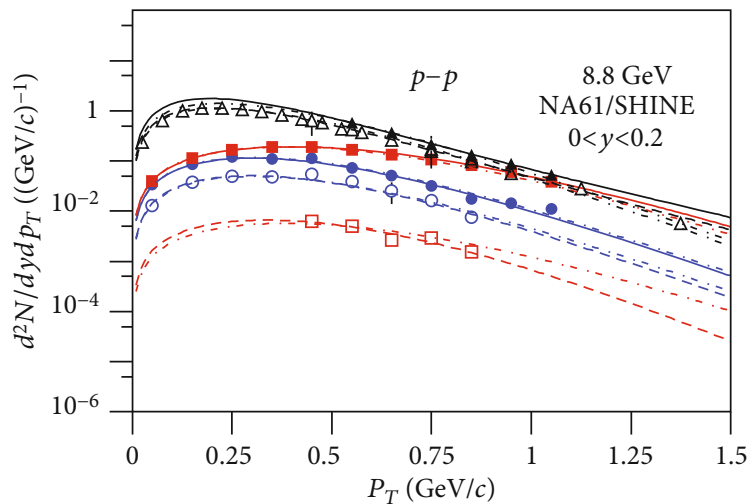

(b)

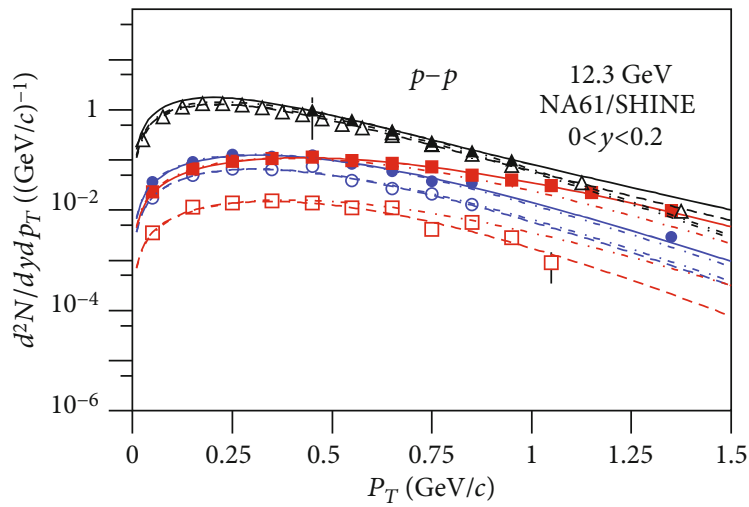

$\pi^{+} \Delta \pi^{-} \Delta$
$K^{+} \bullet K^{-} \circ$
$p=\bar{p} \quad \square$

$\pi^{+} \Delta \pi^{-} \Delta$
$K^{+} \bullet K^{-} \circ$
$p=\bar{p} \square$

(c)

(d)
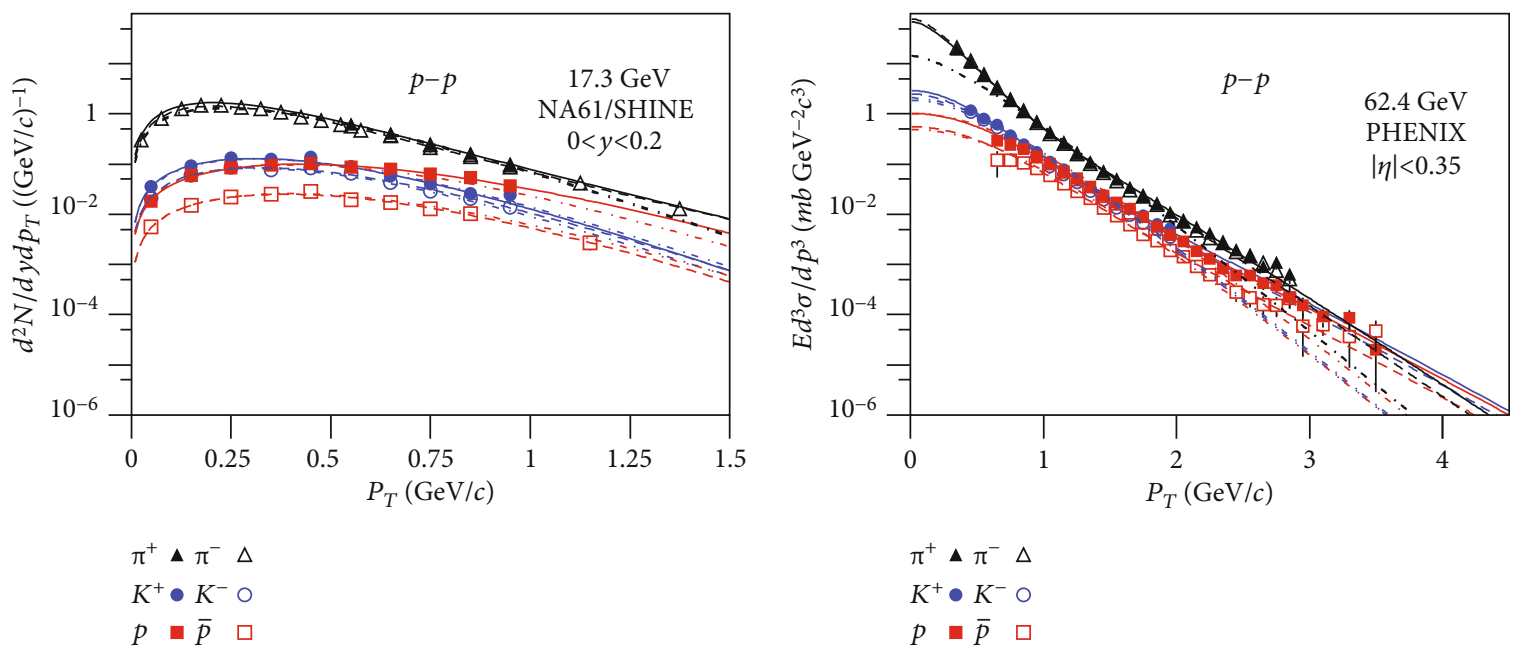

(e)

Figure 3: Continued. 

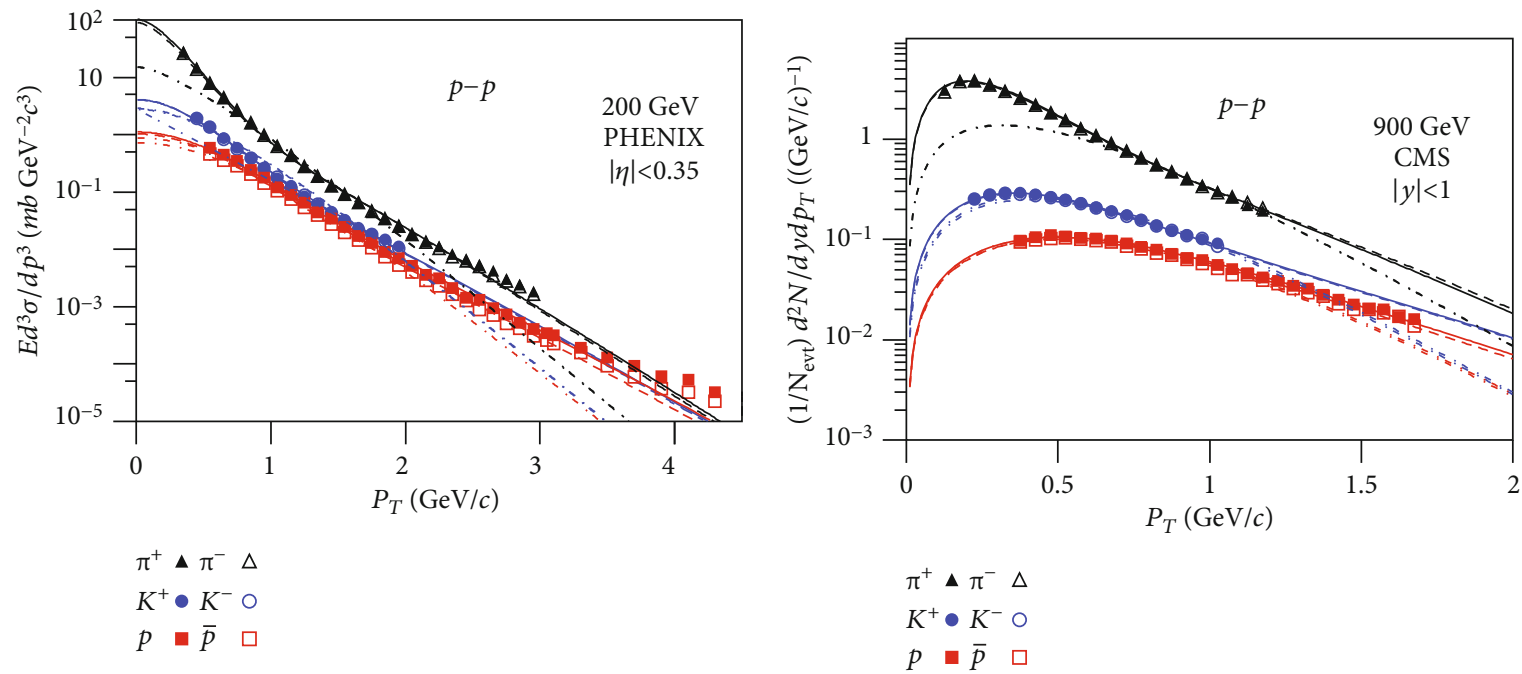

(g)

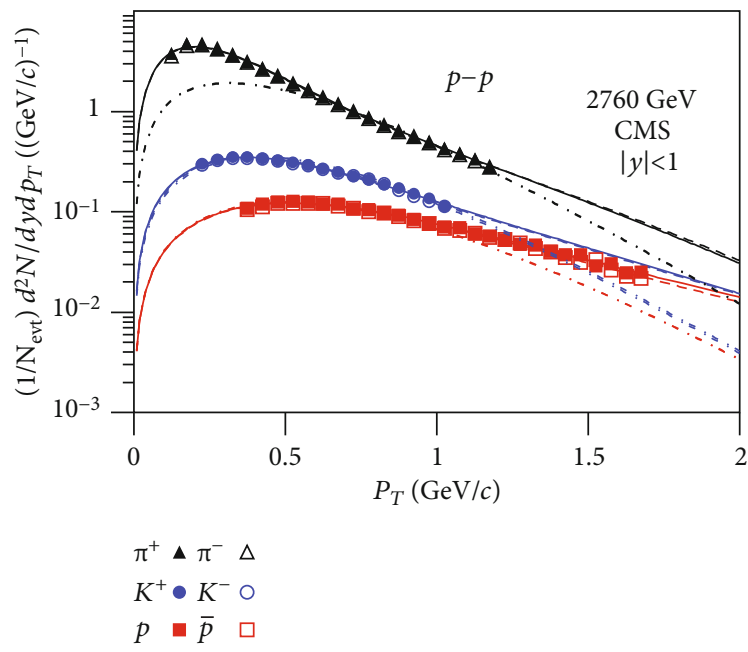

(i)
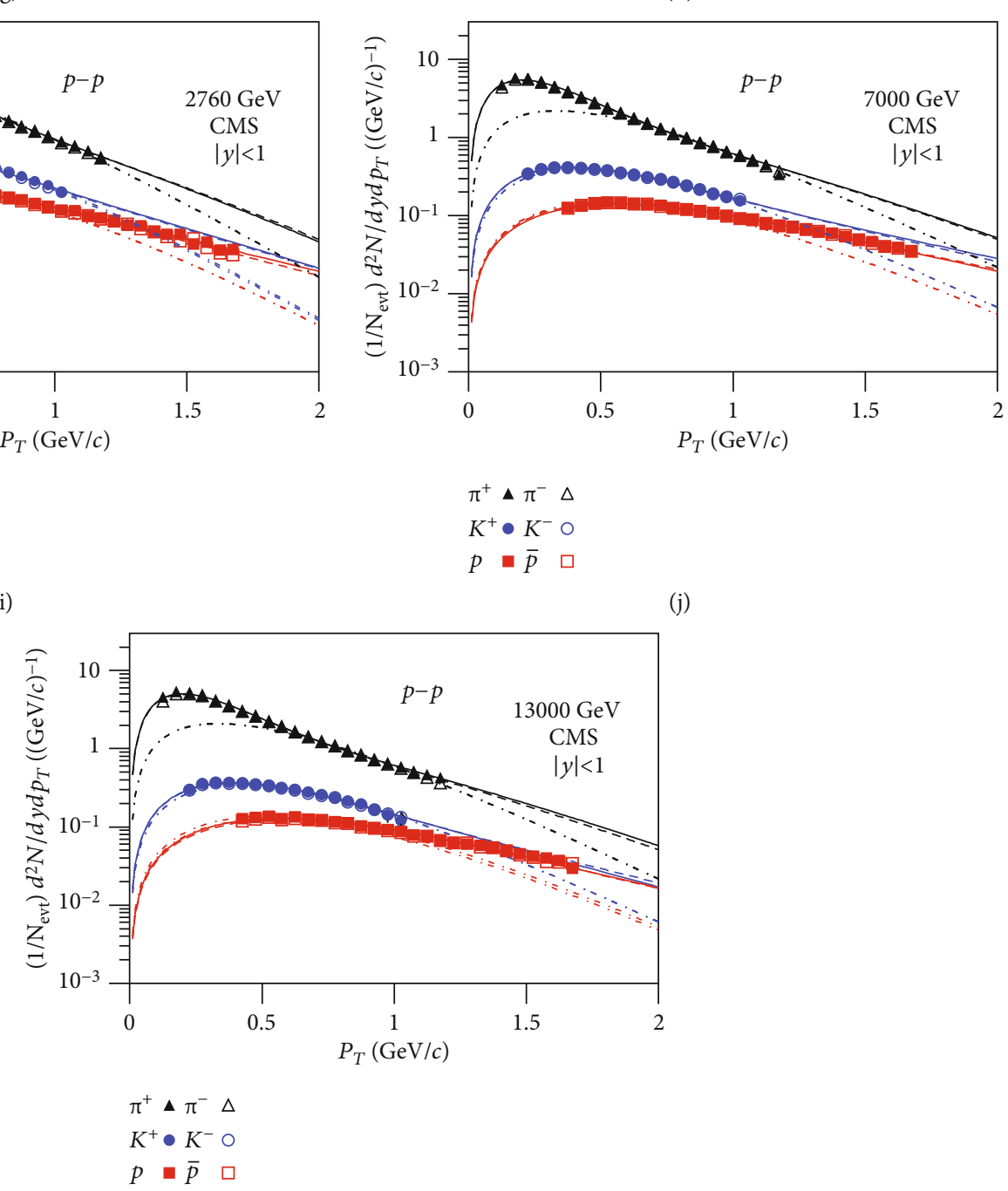

(k)

FIGURE 3: Same as Figure 1 but showing the spectra of various particles produced at mid- $y$ or mid- $\eta$ in INEL $p p$ collisions at high center-ofmass energies $\sqrt{s}$, where the factor $\left(1 / 2 \pi p_{T}\right)$ on the vertical axis is removed and the invariant cross-section $E d^{3} \sigma / d p^{3}$ is used in some cases. The symbols represent the experimental data measured by (a-e) the NA61/SHINE [39, 40], (f, g) the PHENIX [41], and (h-k) the CMS $[42,43]$ Collaborations, where panels $(\mathrm{g}-\mathrm{k})$ appear. 


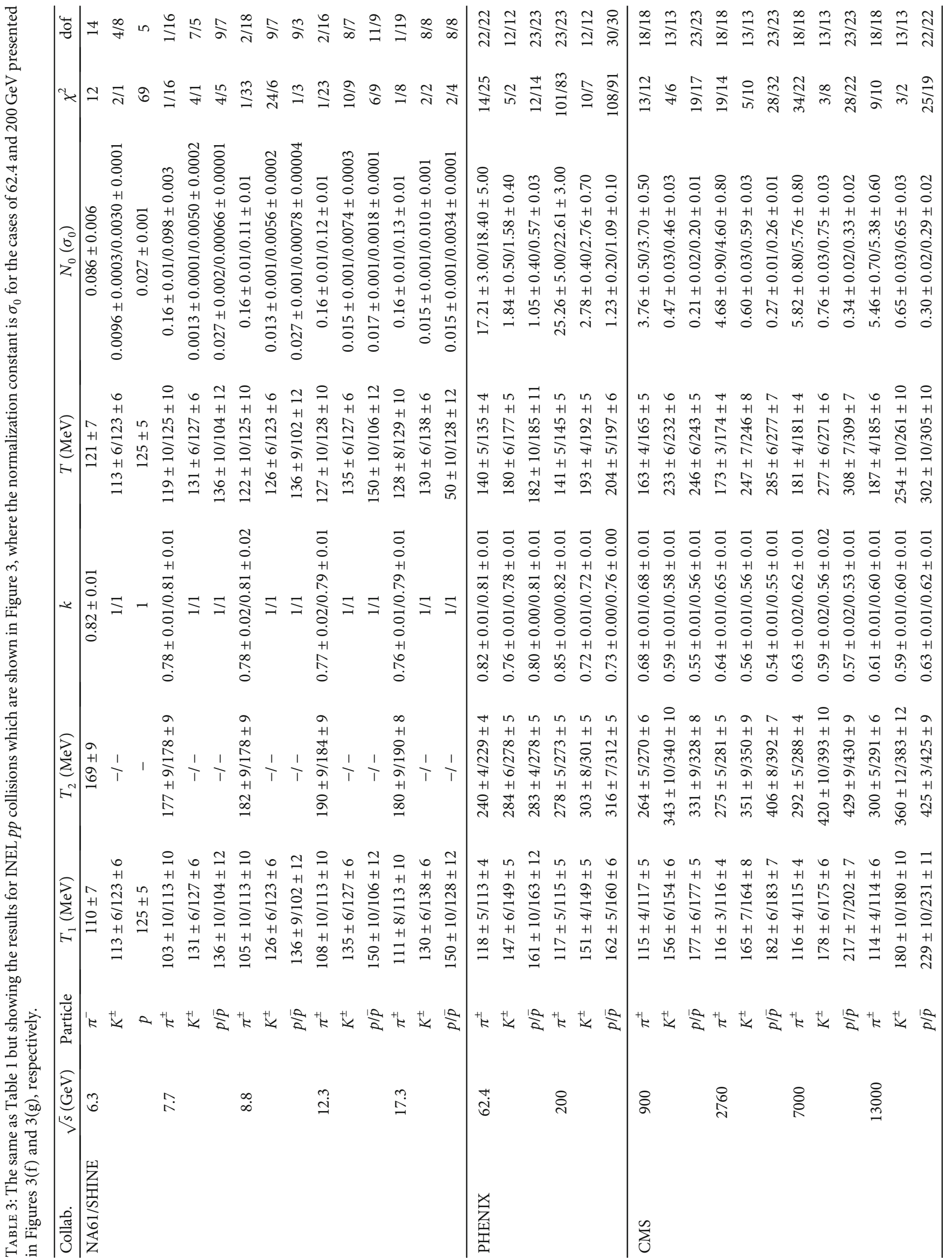




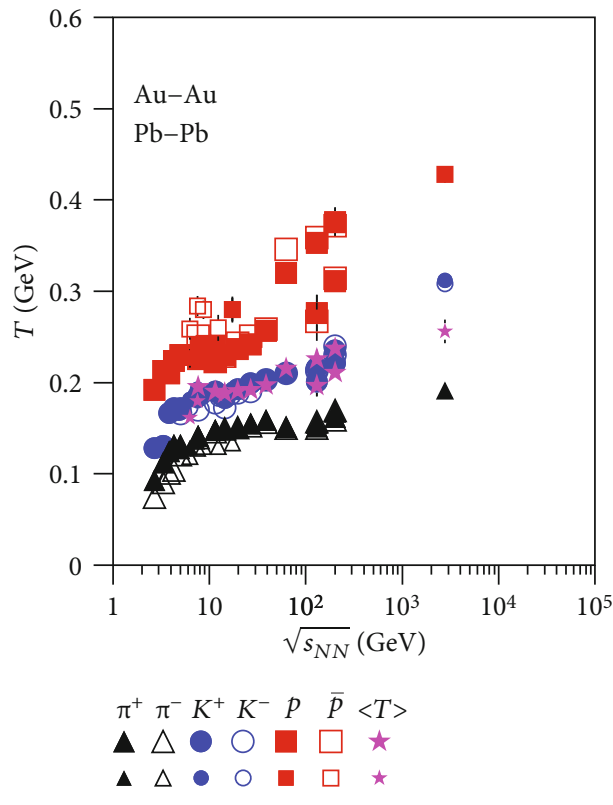

(a)

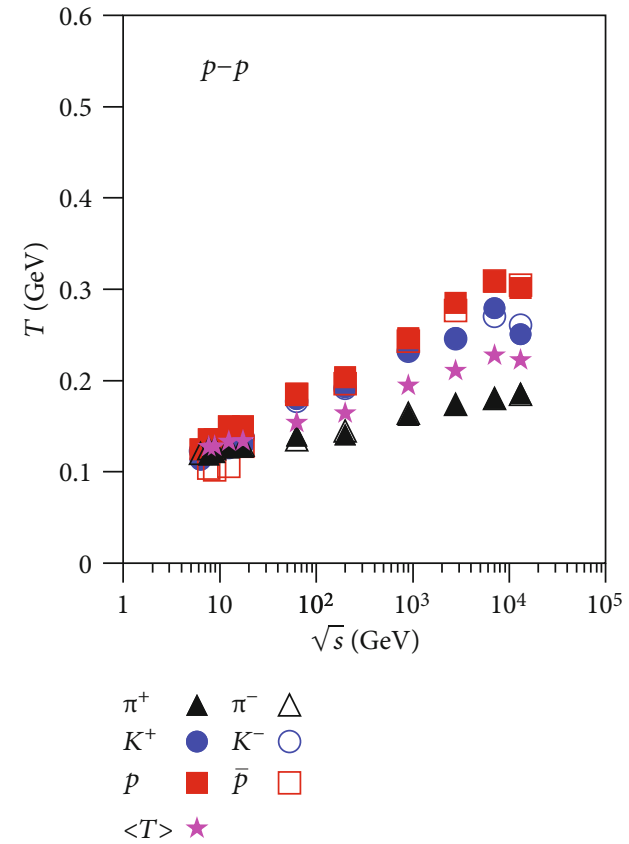

(b)

FIgURE 4: Excitation functions of $T$ and $\langle T\rangle$ in (a) central $\mathrm{Au}-\mathrm{Au}(\mathrm{Pb}-\mathrm{Pb})$ collisions and (b) INEL pp collisions. The symbols corresponding to identified particles are extracted from experimental spectra, where the symbols with small size are used for $\mathrm{Pb}-\mathrm{Pb}$ collisions.

We are not inclined to fit simultaneously the spectra of different particles. Conversely, we use different $T$ for different spectra in this paper.

Figures 5(a) and 5(b) (Figures 5(c) and 5(d)) are similar to Figures 4(a) and 4(b), respectively, but they are showing the excitation functions of $T_{0}\left(\beta_{T}\right)$ and $\left\langle T_{0}\right\rangle \quad\left(\left\langle\beta_{T}\right\rangle\right)$. Figures $6(\mathrm{a})$ and $6(\mathrm{~b})$ are also similar to Figures $4(\mathrm{a})$ and $4(\mathrm{~b})$, respectively, but they are showing the excitation functions of $T_{i}$ and $\left\langle T_{i}\right\rangle$. One can see that $T_{0},\left\langle T_{0}\right\rangle, \beta_{T},\left\langle\beta_{T}\right\rangle, T_{i}$ , and $\left\langle T_{i}\right\rangle$ increase with the increase of $\ln \left(\sqrt{s_{N N}}\right)[\ln (\sqrt{s})]$ in general, and $T_{0}$ for pion emission and $\left\langle T_{0}\right\rangle$ appears in the trend of saturation at the RHIC and LHC. Meanwhile, $T_{0},\left\langle T_{0}\right\rangle, T_{i}$, and $\left\langle T_{i}\right\rangle$ increase, and $\beta_{T}$ and $\left\langle\beta_{T}\right\rangle$ decrease, with the increase of the particle mass.

It is regretful that some particles are absent in experimental measurements at the energies below $10 \mathrm{GeV}$. This renders that $\langle T\rangle,\left\langle T_{0}\right\rangle,\left\langle\beta_{T}\right\rangle$, and $\left\langle T_{i}\right\rangle$ are not available in the energy range of several $\mathrm{GeV}$. From the trends of available $T, T_{0}, \beta_{T}$, and $T_{i}$, we may estimate that $\langle T\rangle,\left\langle T_{0}\right\rangle,\left\langle\beta_{T}\right\rangle$, and $\left\langle T_{i}\right\rangle$ increase (quickly) with the increase of $\ln \left(\sqrt{s_{N N}}\right)[\ln (\sqrt{s})]$ in the energy range of several $\mathrm{GeV}$. In particular, some excitation functions show little peak around $10 \mathrm{GeV}$, which should be studied further in future. Meanwhile, we hope to obtain more data in the energy range of several $\mathrm{GeV}$ in future.

It should be noted that since empirical Equation (18) is essential for obtaining the energy dependence of $T_{0}$ and $\beta_{T}$, it seems that one can obtain arbitrary results by choosing another parametrization of $k_{0}$. In particular, it seems possibly to choose a parametrization of $k_{0}$ so that $\pi^{+}\left(\pi^{-}\right), K^{+}\left(K^{-}\right)$, and $p(\bar{p})$ could have similar $T_{0}$ and $\beta_{T}$ even though the combined temperature $T$ (in Figure 4 ) for these particles are quite different. This case corresponds to the a single scenario for kinetic freeze-out, which is not consistent with two or multiple scenarios observed in other studies [63-66]. We are inclined to use multiple scenarios [67] due to a more accurate fit to the wider $p_{T}$ spectra. To coordinate the single and multiple scenarios, we may regard the multiple scenarios as a refined situation of the single scenario, which will be discussed later in detail.

The trends of excitation functions render that the collision system undergoes different evolution processes. From several $\mathrm{GeV}$ to about $10 \mathrm{GeV}$, the violent degree of collisions increases with the increasing of the energy and matter density of the collision system. The hadronic matter in the collision system stays at a state with an ever higher density and temperature. At about $10 \mathrm{GeV}$, the energy and matter density of the collision system reaches to a high value. The temperature is also high, which is needed to come into notice. At above $10 \mathrm{GeV}$, the energy and matter density of the collision system reaches to a higher value. The temperature is also higher. However, because the phase transition from hadronic matter to QGP had happened possibly, the temperature is limited, which results in the levels of $T_{0}$ for pion emission and $\left\langle T_{0}\right\rangle$ had stabilized.

Before summary and conclusions, we would like to point out that we have used a new method to extract $T_{0}, \beta_{T}$, and $T_{i}$. After fitting the $p_{T}\left(m_{T}\right)$ spectra by using the two-component standard distribution Equations (6) or (7) in which the free parameters are the effective temperatures $T_{1}$ and $T_{2}$ and the contribution fraction $k$ of the first component, the derived parameter, the effective temperature $T$, can be obtained from the weighted average formula Equation (8). 


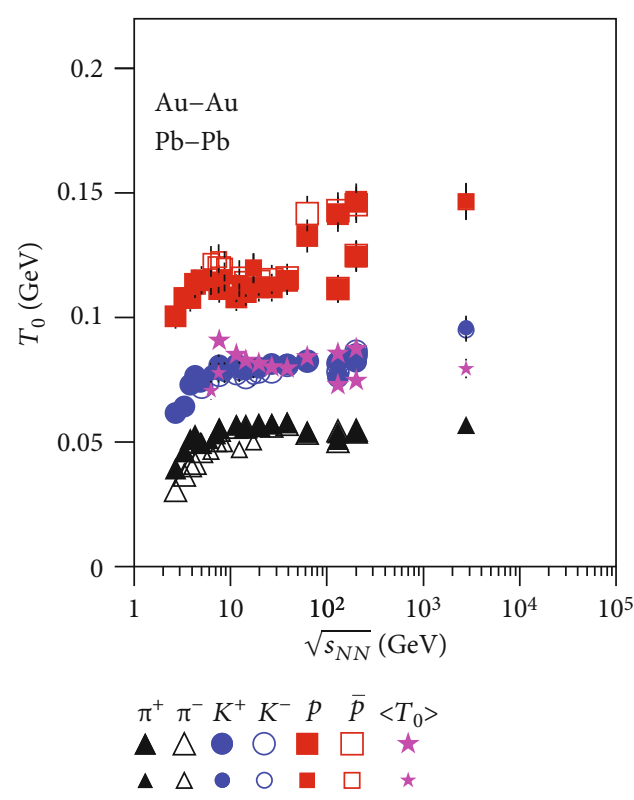

(a)

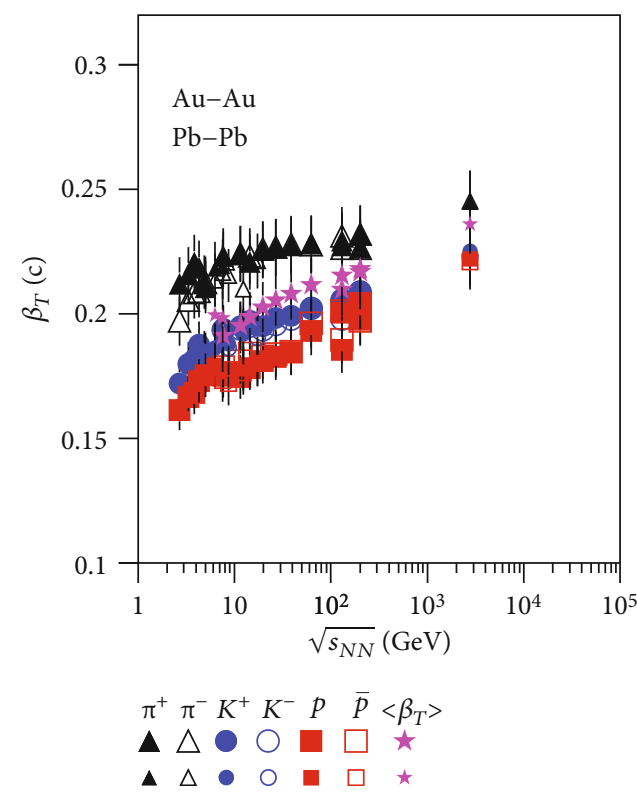

(c)

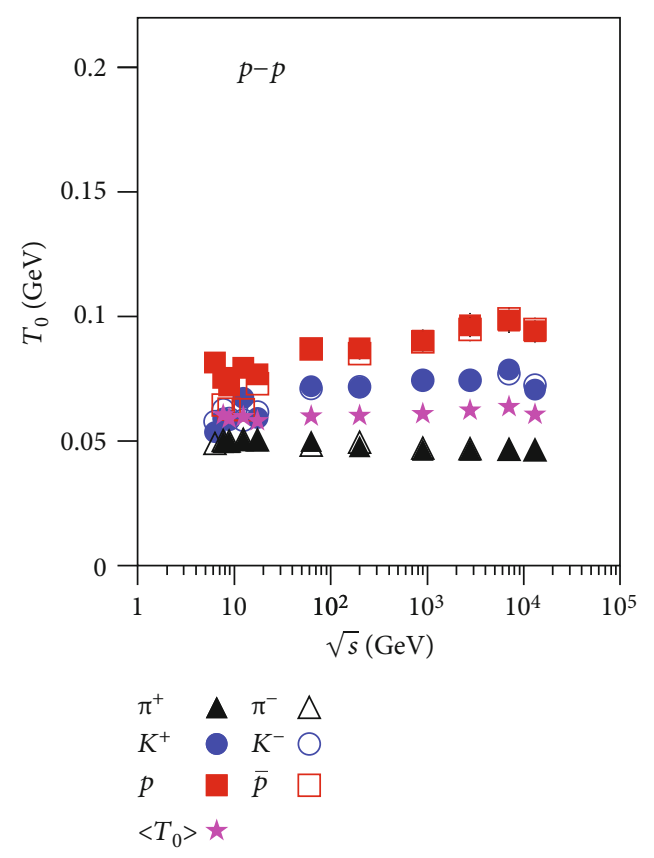

(b)

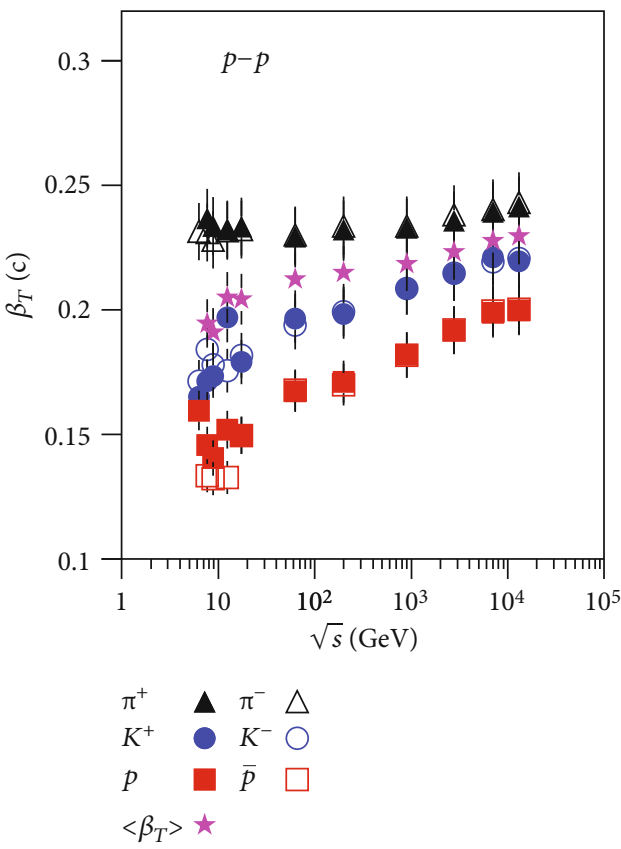

(d)

Figure 5: Same as Figure 4 but showing the excitation functions of (a) and (b) $T_{0}$ and $\left\langle T_{0}\right\rangle$, as well as (c) and (d) $\beta_{T}$ and $\left\langle\beta_{T}\right\rangle$.

Then, the derived parameters, the kinetic freeze-out temperature $T_{0}$, and transverse flow velocity $\beta_{T}$, can be obtained, respectively, from Equations (16) and (17) which are related to the mean transverse momentum $\left\langle p_{T}\right\rangle$. The derived parameter, the initial temperature $T_{i}$, can be obtained from Equation (29) which is related to the root-mean-square $\sqrt{\left\langle p_{T}^{2}\right\rangle}$.

According to the analysis of the spectra of six hadron species listed in Tables 1 and 2, one can see that pions, kaons, and (anti)protons correspond to different temperatures of emission source. This shows a mass-dependent multiple scenario for kinetic freeze-out. Moreover, in AA collisions at energies below the LHC, charged pions can be redistributed between two sources; one is hot and another is cold, while kaons and (anti)protons are located in single (hot) sources (though with different temperatures) in most cases. This is understandable. That charged pions come from resonance decays contribute a relative large fraction in the low- $p_{T}$ region, which can be described by the first component in 


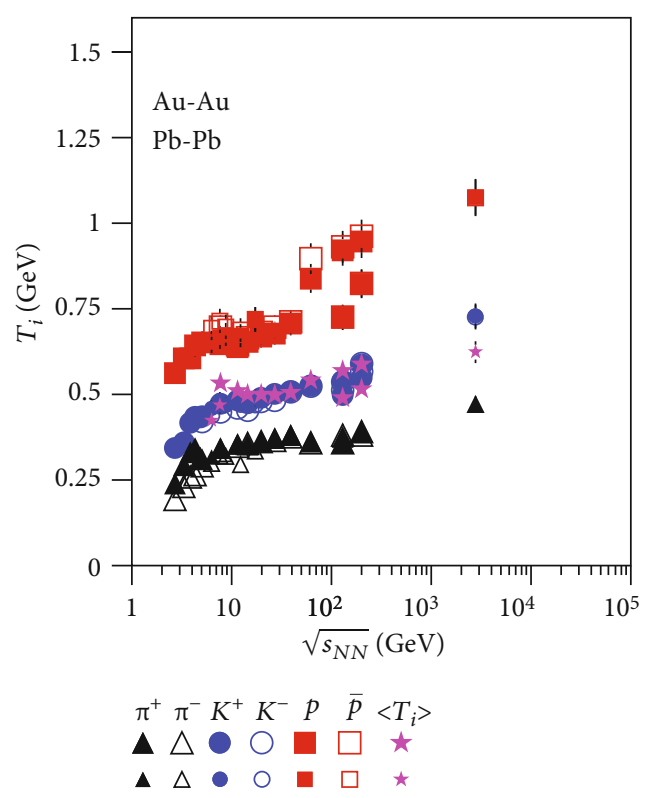

(a)

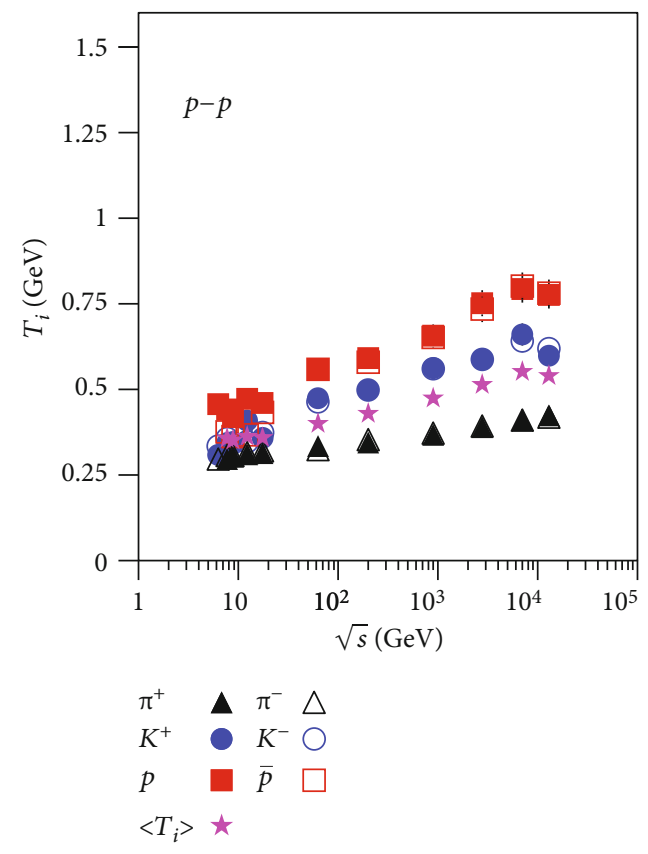

(b)

Figure 6: Same as Figure 4 but showing the excitation functions of $T_{i}$ and $\left\langle T_{i}\right\rangle$.

Equation (6) or (7). Some low- $p_{T}$ pions from nonresonance decay can be also described by the first component. As an ensemble, Equation (1) describes the cold source with low $T$ for all low- $p_{T}$ pions. However, the resonance decays for kaons and (anti)protons are relatively small comparing to those for pions in the low- $p_{T}$ region, which are concealed in a single source.

Naturally, if we regard $\langle T\rangle,\left\langle T_{0}\right\rangle,\left\langle\beta_{T}\right\rangle$, and $\left\langle T_{i}\right\rangle$ as common quantities corresponding to emissions of various hadron species, we may use the mass-independent single scenario for kinetic freeze-out and other system evolution stages such as chemical freeze-out and initial state. It is contentious that the mass-independent single scenario or mass-dependent multiple scenario is right due to different physics thinkings. In our opinion, the mass-independent single scenario is a very ideal situation which is similar to the equilibrium state of mixture gas. And the massdependent multiple scenario describes a refined emission process which "shows massive particles coming out of the system earlier in time with smaller radial flow velocities, which is hydrodynamic behavior" [68]. The temperatures discussed in this paper reflect mainly the kinetic energies of various hadron species but do not have certainly the statistical sense.

We note that, in the mass-dependent multiple scenario for kinetic freeze-out (Figures 4 and 5), the obtained temperature for proton emission is much larger than that for pion emission. This reflects that protons coming out of the system is much earlier than pions due to much larger mass of proton comparing to pion. This phenomenon is a hydrodynamic behavior [68], in which massive particles are early left behind in the evolution process of the collision system. In other words, massive particles are not emitted from the system on their own initiative due to high $T_{0}$ but are left behind under compulsion due to low $\beta_{T}$ and large $m_{0}$. In fact, some protons which existed in projectile and target nuclei appear in rapidity space as leading protons outside the fireball. This issue also results in protons coming out of the system to be earlier than pions.

Because $T_{0}$ and $\beta_{T}$ are model dependent, this paper is different from Figures 37 and 39 in Ref. [32], though this paper is less model dependent and Ref. [32] is much model dependent. In Ref. [32], a flow velocity profile parameter $n$ is used in the extraction of $T_{0}$ and $\beta_{T}$. The parameter $n$ can be largely changed from 0 to 2 in AA collisions and above 4 in pp collisions, which is mutable and debatable. The pion spectra in the low- $p_{T}$ region $(<0.5 \mathrm{GeV} / c)$ are excluded from the fit [32] due to resonance decay, which overrates $T_{0}$ and $\beta_{T}$. Our work shows that $T_{0}\left(\beta_{T}\right)$ in $p p$ collisions is slightly smaller than (almost equal to) those in AA collisions, which is in agreement with our recent work [12] in which the intercept in the linear relation of $T$ versus $m_{0}$ is regarded as $T_{0}$ and the slope in the linear relation of $\left\langle p_{T}\right\rangle$ versus $m_{0} \bar{\gamma}$ is regarded as $\beta_{T}$. This result is understandable due to similar collective behavior as in AA collisions appearing in $p p$ collisions [10].

We would like to emphasize that this paper is a datadriven reanalysis based on some physics considerations, but not a simple fit to the data. From the data-driven reanalysis, the excitation functions of some quantities such as the effective temperature $T$ and its weighted average $\langle T\rangle$, the kinetic freeze-out temperature $T_{0}$ and its weighted 
average $\left\langle T_{0}\right\rangle$, the transverse flow velocity $\beta_{T}$ and its weighted average $\left\langle\beta_{T}\right\rangle$, and the initial temperature $T_{i}$ and its weighted average $\left\langle T_{i}\right\rangle$ have been obtained. These excitation functions have shown some obvious laws with the increase of collision energy.

In the above discussions, to obtain $\bar{\gamma}$ then $\beta_{T}$, we have used the MC method. Figures 5(c) and 5(d) are a direct result by the MC method. As a statistical model is implemented for Figures 1-3, we can also obtain the curves by Equation (19), which is in terms of the MC method. In fact, in the calculation by the MC method, after the shuffled treatment due to the randomicity by the Matlab code, we can obtain a lot of "simulated data". Then, we may count them in different $p_{T}\left(m_{T}-m_{0}\right)$ bins and obtain similar or the same results to the curves in Figures 1-3. In case in the event that the numbers are being not too large, we shall observe fluctuations around the curves. As an example, for $K^{+}$spectra in Figures 1(a)-1(f), the dotted curves and crosses represent the MC results with high and low statistics, respectively. The two results from the analytical function and MC method confirmed each other.

\section{Summary and Conclusion}

We summarize here our main observations and conclusions.

(a) The transverse momentum or mass spectra of $\pi^{+}, \pi^{-}$, $K^{+}, K^{-}, p$, and $\bar{p}$ at mid- $y$ or mid- $\eta$ produced in central $\mathrm{Au}-\mathrm{Au}(\mathrm{Pb}-\mathrm{Pb})$ collisions over an energy range from 2.7 to 200 (6.3 to 2760 ) GeV have been analyzed in this work. Meanwhile, the spectra in INEL $p p$ collisions over an energy range from 6.3 to $13000 \mathrm{GeV}$ have also been analyzed. In most cases, the experimental data measured by the E866, E895, E802, NA49, NA61/SHINE, STAR, PHENIX, ALICE, and CMS Collaborations are approximately fitted by the (two-component) standard distribution in which the temperature concept is the closest to the ideal gas model.

(b) The effective temperature and its excitation function are obtained from the transverse momentum or mass spectra of identified particles produced in collisions at high energies. The kinetic freeze-out temperature and transverse flow velocity and their excitation functions are extracted from the formulas related to the average transverse momentum, which is based on the multisource thermal model. The initial temperature and its excitation function are extracted from the formula related to the root-mean-square transverse momentum, which is based on the color string percolation model.

(c) With the increase of collision energy, the four derived parameters and each average increase (quickly) from a few $\mathrm{GeV}$ to about $10 \mathrm{GeV}$, then increases slowly after $10 \mathrm{GeV}$. In particular, the kinetic freeze-out temperature for pion emission and its average finally appear at the trend of saturation at the RHIC and LHC. Meanwhile, the three derived temperatures increase and the derived transverse flow velocity decrease with the increase of particle mass, which result in a mass-dependent multiple scenario for kinetic freeze-out and other system evolution stages such as chemical freeze-out and initial state.

\section{Data Availability}

The data used to support the findings of this study are included within the article and are cited at relevant places within the text as references.

\section{Ethical Approval}

The authors declare that they are in compliance with ethical standards regarding the content of this paper.

\section{Disclosure}

The funding agencies have no role in the design of the study; in the collection, analysis, or interpretation of the data; in the writing of the manuscript; or in the decision to publish the results.

\section{Conflicts of Interest}

The authors declare that there are no conflicts of interest regarding the publication of this paper.

\section{Acknowledgments}

This work was supported by the National Natural Science Foundation of China under Grant Nos. 11575103 and 11947418, the Chinese Government Scholarship (China Scholarship Council), the Scientific and Technological Innovation Programs of Higher Education Institutions in Shanxi (STIP) under Grant No. 201802017, the Shanxi Provincial Natural Science Foundation under Grant No. 201901D111043, and the Fund for Shanxi "1331 Project" Key Subjects Construction.

\section{References}

[1] S. Gupta, X. Luo, B. Mohanty, H. G. Ritter, and N. Xu, "Scale for the phase diagram of quantum chromodynamics," Science, vol. 332, no. 6037, pp. 1525-1528, 2011.

[2] N. Xu and STAR Collaboration, "An overview of STAR experimental results,” Nuclear Physics A, vol. 931, pp. 1-12, 2014.

[3] A. Andronic, P. Braun-Munzinger, K. Redlich, and J. Stachel, "Decoding the phase structure of QCD via particle production at high energy," Nature, vol. 561, no. 7723, pp. 321-330, 2018.

[4] J. Adams, M. M. Aggarwal, Z. Ahammed et al., "Experimental and theoretical challenges in the search for the quark-gluon plasma: the STAR Collaboration's critical assessment of the evidence from RHIC collisions," Nuclear Physics A, vol. 757, no. 1-2, pp. 102-183, 2005.

[5] K. Adcox, S. S. Adler, S. Afanasiev et al., "Formation of dense partonic matter in relativistic nucleus-nucleus collisions at RHIC: experimental evaluation by the PHENIX Collaboration," Nuclear Physics A, vol. 757, no. 1-2, pp. 184-283, 2005. 
[6] J. F. Grosse-Oetringhaus and Alice Collaboration, "Overview of ALICE results at Quark Matter 2014," Nuclear Physics A, vol. 931, pp. 22-31, 2014.

[7] X. Luo and N. Xu, "Search for the QCD critical point with fluctuations of conserved quantities in relativistic heavy-ion collisions at RHIC: an overview," Nuclear Science and Techniques, vol. 28, no. 8, article 112, 2017.

[8] M. R. Haque, C. Jena, and B. Mohanty, "A review of elliptic flow of light nuclei in heavy-ion collisions at RHIC and LHC energies," Advances in High Energy Physics, vol. 2017, Article ID 1248563, 15 pages, 2017.

[9] S. Bhattacharyya, M. Haiduc, A. T. Neagu, and E. Firu, "A comparative study of ratio in proton-proton collisions at different energies: experimental results versus model simulation," Advances in High Energy Physics, vol. 2018, Article ID 6307205, 7 pages, 2018.

[10] H. Song, Y. Zhou, and K. Gajdošová, "Collective flow and hydrodynamics in large and small systems at the LHC," Nuclear Science and Techniques, vol. 28, no. 7, p. 99, 2017.

[11] S. Schlichting and P. Tribedy, "Collectivity in small collision systems: an initial-state perspective," Advances in High Energy Physics, vol. 2016, Article ID 8460349, 17 pages, 2016.

[12] H.-L. Lao, F.-H. Liu, B.-C. Li, M.-Y. Duan, and R. A. Lacey, "Examining the model dependence of the determination of kinetic freeze-out temperature and transverse flow velocity in small collision system," Nuclear Science and Techniques, vol. 29, no. 11, p. 164, 2018.

[13] H.-L. Lao, F.-H. Liu, B.-C. Li, and M.-Y. Duan, "Kinetic freezeout temperatures in central and peripheral collisions: which one is larger?," Nuclear Science and Techniques, vol. 29, no. 6 , p. $82,2018$.

[14] J. Cleymans, "The physics case for the $\sqrt{s_{N N}} \approx 10 \mathrm{GeV}$ energy region," in Walter Greiner Memorial Volume, P. O. Hess, Ed., World Scientiflc, Singapore, 2018, https://arxiv.org/abs/1711 .02882 .

[15] A. Andronic, P. Braun-Munzinger, and J. Stachel, "Thermal hadron production in relativistic nuclear collisions," Acta Physica Polonica B, vol. 40, pp. 1005-1012, 2009.

[16] A. Andronic, P. Braun-Munzinger, and J. Stachel, "The horn, the hadron mass spectrum and the QCD phase diagram the statistical model of hadron production in central nucleus-nucleus collisions," Nuclear Physics A, vol. 834, no. $1-4$, pp. 237c-240c, 2010.

[17] J. Cleymans, H. Oeschler, K. Redlich, and S. Wheaton, "Comparison of chemical freeze-out criteria in heavy-ion collisions," Physical Review C, vol. 73, no. 3, article 034905, 2006.

[18] A. Andronic, P. Braun-Munzinger, and J. Stachel, "Hadron production in central nucleus-nucleus collisions at chemical freeze-out," Nuclear Physics A, vol. 772, no. 3-4, pp. 167-199, 2006.

[19] F.-H. Liu, Y.-Q. Gao, T. Tian, and B.-C. Li, "Unified description of transverse momentum spectrums contributed by soft and hard processes in high-energy nuclear collisions," The European Physical Journal A, vol. 50, no. 6, p. 94, 2014.

[20] L.-N. Gao, F.-H. Liu, and R. A. Lacey, "Excitation functions of parameters in Erlang distribution, Schwinger mechanism, and Tsallis statistics in RHIC BES program," The European Physical Journal A, vol. 52, no. 5, p. 137, 2016.

[21] W.-J. Xie, "Transverse momentum spectra in high-energy nucleus-nucleus, proton-nucleus and proton-proton collisions," Chinese Physics C, vol. 35, no. 12, pp. 1111-1119, 2011.
[22] L. J. Gutay, A. S. Hirsch, R. P. Scharenberg, B. K. Srivastava, and C. Pajares, "De-confinement in small systems: clustering of color sources in high multiplicity $\bar{p} p$ collisions at $\sqrt{s}=1.8$ TeV," International Journal of Modern Physics E, vol. 24, no. 12, article 1550101, 2015.

[23] R. . P. Scharenberg, B. . K. Srivastava, and C. Pajares, "Exploring the initial stage of high multiplicity proton-proton collisions by determining the initial temperature of the quarkgluon plasma," Physical Review D, vol. 100, no. 11, article 114040, 2019.

[24] P. Sahoo, S. De, S. K. Tiwari, and R. Sahoo, "Energy and centrality dependent study of deconfinement phase transition in a color string percolation approach at RHIC energies," The European Physical Journal A, vol. 54, no. 8, p. 136, 2018.

[25] L. Ahle, Y. Akiba, K. Ashktorab et al., "Excitation function of $\mathrm{K}^{+}$and $\pi^{+}$production in $\mathrm{Au}+\mathrm{Au}$ reactions at 2-10 $\mathrm{A} \mathrm{GeV}$," Physics Letters B, vol. 476, no. 1-2, pp. 1-8, 2000.

[26] J. L. Klay, N. N. Ajitanand, J. M. Alexander et al., "Longitudinal flow of protons from (2-8)A GeV central $A u+A u$ collisions," Physical Review Letters, vol. 88, no. 10, article 102301, 2002.

[27] J. L. Klay, N. N. Ajitanand, J. M. Alexander et al., "Charged pion production in $2 A$ to $8 A \mathrm{GeV}$ central $A u+A u$ collisions," Physical Review C, vol. 68, no. 5, article 054905, 2003.

[28] L. Ahle, Y. Akiba, K. Ashktorab et al., "Kaon production in Au + Au collisions at $11.6 \mathrm{~A} \mathrm{GeV/c,"} \mathrm{Physical} \mathrm{Review} \mathrm{C,} \mathrm{vol.} \mathrm{58,}$ no. 6, pp. 3523-3538, 1998.

[29] L. Ahle, Y. Akiba, K. Ashktorab et al., "Particle production at high baryon density in central $\mathrm{Au}+\mathrm{Au}$ reactions at 11.6A GeV/c," Physical Review C, vol. 57, no. 2, pp. R466R470, 1998.

[30] L. Adamczyk, J. K. Adkins, G. Agakishiev et al., "Bulk properties of the medium produced in relativistic heavy-ion collisions from the beam energy scan program," Physical Review C, vol. 96, no. 4, article 044904, 2017.

[31] V. Bairathi and for the STAR Collaboration, "Study of the bulk properties of the system formed in $\mathrm{Au}+\mathrm{Au}$ collisions at $\sqrt{s_{N N}}=14.5 \mathrm{GeV}$ using the STAR detector at RHIC," Nuclear Physics A, vol. 956, pp. 292-295, 2018.

[32] B. I. Abelev, M. M. Aggarwal, Z. Ahammed et al., "Systematic measurements of identified particle spectra in pp, $d+A u$, and $\mathrm{Au}+\mathrm{Au}$ collisions at the STAR detector," Physical Review C, vol. 79, no. 3, article 034909, 2009.

[33] K. Adcox, S. S. Adler, N. N. Ajitanand et al., "Centrality dependence of $\pi^{+} / \pi^{-}, \mathrm{K}^{+} / \mathrm{K}^{-}, p$, and $\bar{p}$ production from $\sqrt{s_{N N}}=130$ $\mathrm{GeV} \mathrm{Au}+\mathrm{Au}$ collisions at RHIC," Physical Review Letters, vol. 88, no. 24, article 242301, 2002.

[34] S. S. Adler, S. Afanasiev, C. Aidala et al., "Identified charged particle spectra and yields in $\mathrm{Au}+\mathrm{Au}$ collisions at $\sqrt{s_{N N}}=$ 200 GeV," Physical Review C, vol. 69, no. 3, article 034909, 2004.

[35] C. Alt, T. Anticic, B. Baatar et al., "Energy and centrality dependence of $\bar{p}$ and $p$ production and the $\bar{\Lambda} / \bar{p}$ ratio in $\mathrm{Pb}$ $+\mathrm{Pb}$ collisions between $20 \mathrm{AGeV}$ and $158 \mathrm{AGeV}$," Physical Review C, vol. 73, no. 4, article 044910, 2006.

[36] C. Alt, T. Anticic, B. Baatar et al., "Pion and kaon production in central $\mathrm{Pb}+\mathrm{Pb}$ collisions at $20 \mathrm{~A}$ and $30 \mathrm{~A} \mathrm{GeV}$ : evidence for the onset of deconfinement," Physical Review C, vol. 77, no. 2, article 024903, 2008.

[37] S. V. Afanasiev, T. Anticic, D. Barna et al., "Energy dependence of pion and kaon production in central $\mathrm{Pb}+\mathrm{Pb}$ collisions," Physical Review C, vol. 66, no. 5, article 054902, 2002. 
[38] B. Abelev, J. Adam, D. Adamová et al., "Centrality dependence of $\pi, K$, and $p$ production in $\mathrm{Pb}-\mathrm{Pb}$ collisions at $\sqrt{s_{N N}}=2.76$ TeV," Physical Review C, vol. 88, no. 4, article 044910, 2013.

[39] N. Abgrall, A. Aduszkiewicz, Y. Ali et al., "Measurement of negatively charged pion spectra in inelastic $\mathrm{p}+\mathrm{p}$ interactions at $p_{\text {lab }}=20,31,40,80$ and $158 \mathrm{GeV} / c$," The European Physical Journal C, vol. 74, no. 3, article 2794, 2014.

[40] A. Aduszkiewicz, Y. Ali, E. Andronov et al., "Measurements of $\pi^{ \pm}, \mathrm{K}^{ \pm}, p$ and $\bar{p}$ spectra in proton-proton interactions at 20,31 , 40,80 and $158 \mathrm{GeV} / c \mathrm{GeV} / c$ with the NA61/SHINE spectrometer at the CERN SPS," The European Physical Journal C, vol. 77, no. 10, p. 671, 2017.

[41] A. Adare, S. Afanasiev, C. Aidala et al., "Identified charged hadron production in $p+p$ collisions at $\sqrt{s}=200$ and 62.4 GeV," Physical Review C, vol. 83, no. 6, article 064903, 2011.

[42] The CMS Collaboration, S. Chatrchyan, V. Khachatryan et al., "Study of the inclusive production of charged pions, kaons, and protons in $p p$ collisions at $\sqrt{s}=0.9,2.76$, and $7 \mathrm{TeV}$," The European Physical Journal C, vol. 72, article 2164, 2012.

[43] A. M. Sirunyan, A. Tumasyan, W. Adam et al., "Measurement of charged pion, kaon, and proton production in protonproton collisions at $\sqrt{s}=13 \mathrm{TeV}$," Physical Review D, vol. 96, no. 11, article 112003, 2017.

[44] J. Cleymans and D. Worku, "Relativistic thermodynamics: transverse momentum distributions in high-energy physics," The European Physical Journal A, vol. 48, no. 11, p. 160, 2012.

[45] C. Tsallis, "Possible generalization of Boltzmann-Gibbs statistics," Journal of Statistical Physics, vol. 52, no. 1-2, pp. 479487, 1988.

[46] T. S. Biró, G. Purcsel, and K. Ürmössy, "Non-extensive approach to quark matter," The European Physical Journal A, vol. 40, no. 3, p. 325, 2009.

[47] H. Zheng and L. Zhu, "Comparing the Tsallis distribution with and without thermodynamical description in $p+p$ Collisions," Advances in High Energy Physics, vol. 2016, Article ID 9632126, 10 pages, 2016.

[48] J. Schwinger, "On gauge invariance and vacuum polarization," Physical Review, vol. 82, no. 5, pp. 664-679, 1951.

[49] R.-C. Wang and C.-Y. Wong, "Finite-size effect in the Schwinger particle-production mechanism," Physical Review D, vol. 38, no. 1, pp. 348-359, 1988.

[50] C.-Y. Wong, Introduction to High Energy Heavy Ion Collisions, World Scientific, Singapore, 1994.

[51] P. Braun-Munzinger, K. Redlich, and J. Stachel, "Particle production in heavy ion collisions," in Quark-Gluon Plasma 3, R. C. Hwa and X.-N. Wang, Eds., World Scientific, Singapore, 2004, https://arxiv.org/abs/nucl-th/0304013.

[52] E. Schnedermann, J. Sollfrank, and U. Heinz, "Thermal phenomenology of hadrons from $200 \mathrm{~A} \mathrm{GeV} \mathrm{S+S} \mathrm{collisions,"} \mathrm{Phys-}$ ical Review C, vol. 48, no. 5, pp. 2462-2475, 1993.

[53] B. I. Abelev, M. M. Aggarwal, Z. Ahammed et al., "Identified particle production, azimuthal anisotropy, and interferometry measurements in Au+Au collisions at $\sqrt{s_{N N}}=9.2 \mathrm{GeV}$," Physical Review C, vol. 81, no. 2, 2010.

[54] Z. Tang, Y. Xu, L. Ruan, G. van Buren, F. Wang, and Z. Xu, "Spectra and radial flow in relativistic heavy ion collisions with Tsallis statistics in a blast-wave description," Physical Review C, vol. 79, no. 5, article 051901, 2009.

[55] Z.-B. Tang, L. Yi, L. J. Ruan et al., "The Statistical origin of constituent-quark scaling in QGP hadronization," Chinese Physics Letters, vol. 30, no. 3, article 031201, p. 031201, 2013.
[56] K. Jiang, Y. Zhu, W. Liu et al., "Onset of radial flow in $p+p$ collisions,” Physical Review C, vol. 91, no. 2, article 024910, 2015.

[57] R. Hagedorn, "Multiplicities, $p_{T}$ distributions and the expected hadron $\rightarrow$ quark-gluon phase transition," La Rivista del Nuovo Cimento, vol. 6, no. 10, pp. 1-50, 1983.

[58] P. Koch, J. Rafelski, and W. Greiner, "Strange hadrons in hot nuclear matter," Physics Letters B, vol. 123, no. 3-4, pp. 151154, 1983.

[59] P. Braun-Munzinger, D. Magestro, K. Redlich, and J. Stachel, "Hadron production in Au-Au collisions at RHIC," Physics Letters B, vol. 518, no. 1-2, pp. 41-46, 2001.

[60] J.-Y. Ollitrault, "Revealing QCD thermodynamics in ultrarelativistic nuclear collisions," in Proc. 28th Int. Conf. Ultrarelativisitc nucleus-nucleus collisions (quark matter 2019), Wuhan, China, November 2019Nuclear Physics A, to appear, 2020 .

[61] Y.-H. Chen and F.-H. Liu, "Event patterns extracted from anisotropic spectra of charged particles produced in $\mathrm{Pb}-\mathrm{Pb}$ collisions at $2.76 \mathrm{TeV}$," The European Physical Journal A, vol. 53, no. 11, p. 230, 2017.

[62] H.-X. Zhang and P.-J. Shan, "Statistical simulation method for determinating the errors of fit parameters," in Proc. 8th Natl. Conf. Nucl. Phys. (the next volume), Xi'an, China, December $1991 \mathrm{http}: / / \mathrm{cpfd} . c n k i . c o m . c n / A r t i c l e / C P F D T O T A L-$ HWLX199112002128.htm.

[63] S. Chatterjee, S. Das, L. Kumar et al., "Freeze-out parameters in heavy-ion collisions at AGS, SPS, RHIC, and LHC energies," Advances in High Energy Physics, vol. 2015, Article ID 349013, 20 pages, 2015.

[64] S. Chatterjee, B. Mohanty, and R. Singh, "Freezeout hypersurface at energies available at the CERN Large Hadron Collider from particle spectra: flavor and centrality dependence," Physical Review C, vol. 92, no. 2, article 024917, 2015.

[65] S. Chatterjee and B. Mohanty, "Production of light nuclei in heavy-ion collisions within a multiple-freezeout scenario," Physical Review C, vol. 90, no. 3, article 034908, 2014.

[66] D. Thakur, S. Tripathy, P. Garg, R. Sahoo, and J. Cleymans, "Indication of a differential freeze-out in proton-proton and heavy-ion collisions at RHIC and LHC energies," Advances in High Energy Physics, vol. 2016, Article ID 4149352, 13 pages, 2016.

[67] H.-L. Lao, H. R. Wei, F. H. Liu, and R. A. Lacey, “An evidence of mass-dependent differential kinetic freeze-out scenario observed in $\mathrm{Pb}-\mathrm{Pb}$ collisions at $2.76 \mathrm{TeV}$," The European Physical Journal A, vol. 52, no. 7, p. 203, 2016.

[68] R. Sahoo, "Possible formation of QGP-droplets in protonproton collisions at the CERN Large Hadron Collider," AAPPS Bulletin, vol. 29, no. 4, pp. 16-21, 2019. 